\title{
Correlating Resting-State Functional Magnetic Resonance Imaging Connectivity by Independent Component Analysis-Based Epileptogenic Zones with Intracranial Electroencephalogram Localized Seizure Onset Zones and Surgical Outcomes in Prospective Pediatric Intractable Epilepsy Study
}

\author{
Varina L. Boerwinkle, ${ }^{1,2, \star}$ Deepankar Mohanty, ${ }^{2, \dagger}$ Stephen T. Foldes, ${ }^{3}$ Danielle Guffey, \\ Charles G. Minard, Aditya Vedantam, Jeffrey S. Raskin, Sandi Lam, Margaret Bond, \\ Lucia Mirea, P. David Adelson, ${ }^{1,7}$ Angus A. Wilfong, ${ }^{1,2, \$}$ and Daniel J. Curry ${ }^{5}$
}

\begin{abstract}
The purpose of this study was to prospectively investigate the agreement between the epileptogenic zone(s) (EZ) localization by resting-state functional magnetic resonance imaging (rs-fMRI) and the seizure onset zone(s) (SOZ) identified by intracranial electroencephalogram (ic-EEG) using novel differentiating and ranking criteria of rsfMRI abnormal independent components (ICs) in a large consecutive heterogeneous pediatric intractable epilepsy population without an a priori alternate modality informing EZ localization or prior declaration of total SOZ number. The EZ determination criteria were developed by using independent component analysis (ICA) on rs-fMRI in an initial cohort of 350 pediatric patients evaluated for epilepsy surgery over a 3-year period. Subsequently, these rs-fMRI EZ criteria were applied prospectively to an evaluation cohort of 40 patients who underwent ic-EEG for SOZ identification. Thirty-seven of these patients had surgical resection/disconnection of the area believed to be the primary source of seizures. One-year seizure frequency rate was collected postoperatively. Among the total 40 patients evaluated, agreement between rs-fMRI EZ and ic-EEG SOZ was 90\% (36/40; 95\% confidence interval [CI], 0.76-0.97). Of the 37 patients who had surgical destruction of the area believed to be the primary source of seizures, $27(73 \%)$ rs-fMRI EZ could be classified as true positives, $7(18 \%)$ false positives, and $2(5 \%)$ false negatives. Sensitivity of rs-fMRI EZ was 93\% (95\% CI 78-98\%) with a positive predictive value of 79\% (95\% CI, 63-89\%). In those with cryptogenic localization-related epilepsy, agreement between rs-fMRI EZ and ic-EEG SOZ was 89\% (8/9; 95\% CI, 0.52-99), with no statistically significant difference between the agreement in the cryptogenic and symptomatic localization-related epilepsy subgroups. Two children with negative ic-EEG had removal of the rs-fMRI EZ and were seizure free 1 year postoperatively. Of the 33 patients where at least 1 rs-fMRI EZ agreed with the ic-EEG SOZ, 24\% had at least 1 additional rs-fMRI EZ outside the resection area. Of these patients with un-resected rsfMRI EZ, 75\% continued to have seizures 1 year later. Conversely, among $75 \%$ of patients in whom rs-fMRI agreed with ic-EEG SOZ and had no anatomically separate rs-fMRI EZ, only $24 \%$ continued to have seizures 1 year later. This relationship between extraneous rs-fMRI EZ and seizure outcome was statistically significant $(p=0.01)$.

\footnotetext{
${ }^{1}$ Division of Pediatric Neurology, Barrow Neurological Institute at Phoenix Children's Hospital, Phoenix, Arizona.

${ }^{2}$ Department of Pediatric Neurology, Texas Children's Hospital, Baylor College of Medicine, Houston, Texas.

${ }^{3}$ Neuroscience Research, Barrow Neurological Institute at Phoenix Children's Hospital, Phoenix, Arizona.

${ }^{4}$ Dan L. Duncan Institute for Clinical and Translational Research, Baylor College of Medicine, Houston, Texas.

${ }^{5}$ Department of Pediatric Neurosurgery, Texas Children's Hospital, Baylor College of Medicine, Houston, Texas.

${ }^{6}$ Department of Research, Phoenix Children's Hospital, Phoenix, Arizona.

${ }^{7}$ Division of Pediatric Neurosurgery, Barrow Neurological Institute at Phoenix Children's Hospital, Phoenix, Arizona.

*Present address: Medical Director of Neuro-Critical Care and Resting State fMRI for Epilepsy Surgical Evaluation at the Barrow Neurological Institute at Phoenix Children's Hospital, Phoenix, Arizona.

${ }^{\dagger}$ Present address: Pediatric Neurology Resident at the Monroe Carrell Jr. Children's Hospital at Vanderbilt University, Nashville, Tennessee.

tPresent address: Division Chief of Pediatric Neurology at the Barrow Neurological Institute at Phoenix Children's Hospital, Phoenix, Arizona.

(c) Varina L. Boerwinkle et al. 2017; Published by Mary Ann Liebert, Inc. This is an Open Access article distributed under the terms of the Creative Commons Attribution License, which permits unrestricted use, distribution, and reproduction in any medium, provided the original work is properly cited.
} 
rs-fMRI EZ surgical destruction showed significant association with postoperative seizure outcome. The pediatric population with intractable epilepsy studied prospectively provides evidence for use of resting-state ICA ranking criteria, to identify rs-fMRI EZ, as developed by the lead author (V.L.B.). This is a high yield test in this population, because no seizure nor particular interictal epilepiform activity needs to occur during the study. Thus, rs-fMRI EZ detected by this technique are potentially informative for epilepsy surgery evaluation and planning in this population. Independent of other brain function testing modalities, such as simultaneous EEG-fMRI or electrical source imaging, contextual ranking of abnormal ICs of rs-fMRI localized EZs correlated with the gold standard of SOZ localization, ic-EEG, across the broad range of pediatric epilepsy surgery candidates, including those with cryptogenic epilepsy.

Keywords: epilepsy surgery; functional connectivity; independent component analysis; intraoperative electrocorticography; resting-state functional MRI

\section{Introduction}

I $\mathrm{T}$ IS NOT yet established as to whether resting-state functional magnetic resonance imaging (rs-fMRI) connectivity measures alone can reliably localize the epileptogenic zone (EZ) with the level of certainty needed for surgical resection. Prior studies have evaluated rs-fMRI-derived EZs by comparing them with intracranial electroencephalogram (ic-EEG) data though the sample sizes have been relatively small (Negishi et al., 2011; Stufflebeam et al., 2011; Zhang et al., 2014). In the largest study to date, a $72 \%$ concordance was observed between a developed functional connectivity measure and EZ in 23 patients with focal epilepsy (Lee et al., 2014). In addition to the variability in techniques used to analyze rs-fMRI data, the most abnormal brain area identified by the analysis was the primary factor evaluated to identify the proposed EZ in prior studies. By focusing too narrowly on a single region with the most abnormal connectivity, other relevant EZs and secondary EZs may not be revealed. This potential weakness is further amplified when analyzing data from a heterogeneous pediatric epilepsy population, since the blood-oxygen-level-dependent (BOLD) hemodynamic alterations across the entire brain may reveal additional key elements that are relevant to surgical planning.

Independent component analysis (ICA) is an established data-driven approach for analyzing rs-fMRI that does not have pretest assumptions for areas of ictal dysfunction or seizure propagation. ICA produces spatial brain maps (independent components-ICs; see Supplementary Figs. S1-S3 [Supplementary Data are available online at www.liebertpub .com/brain] for examples of normal, noise, and epileptogenicrelated ICA neuronal ICs) that can describe the participation of a seizure focus in more than one network (Beckmann et al., 2005), thereby highlighting abnormal networks from different regions of the brain. However, selecting the meaningful subset of IC derived from ICA remains a challenge, and the interpretation of the data requires expert review (De Martino et al., 2007). Criteria to distinguish noise from meaningful ICs have been developed by using normal individuals or adult patients, and with the help of supplementary EEG data (Hunyadi et al., 2015; Zhang et al., 2015). Currently, there are no ICA criteria defined for the pediatric epilepsy population, and no study has evaluated the role of rs-fMRI to identify EZs independent of EEG data.

In this study, we aimed at applying ICA criteria developed from studying rs-fMRIs in a pediatric epilepsy population to identify rs-fMRI EZs independent of EEG data. Subsequently, by comparing the prospectively determined rs-fMRI EZs with ic-EEG-identified seizure onset zones (SOZs), we intended to demonstrate the reliability of ICA criteria from rs-fMRI as a clinically relevant, noninvasive modality for EZ localization in pediatric epilepsy.

\section{Methods \\ Study design}

Developmental phase. Within a consecutive series of pediatric patients with intractable epilepsy due to various etiologies including symptomatic and cryptogenic epilepsy (Table 1), a total of 350 children (ages 18 months to 19.8 years) with intractable epilepsy received rs-fMRI as part of their standard clinical MRI at Texas Children's Hospital from June 2012 to April 2015. Approval was obtained by the local institutional review board (IRB H-32492), with the determination that since rs-

Table 1. Patient Characteristics

\begin{tabular}{lc}
\hline & $\begin{array}{c}\text { All patients } \\
(\mathrm{N}=40)\end{array}$ \\
\hline Age (years), mean $(S D), N=39$ & $11.6(5.3)$ \\
Male, $N(\%)$ & $23(58)$ \\
Handedness, $N(\%), N=35$ & \\
Left & $5(14)$ \\
Right & $27(77)$ \\
Other & $3(9)$ \\
Seizure classification, $N(\%)$ & \\
CLRE & $9(23)$ \\
SLRE & $31(78)$ \\
Primary etiology, $N(\%)$ & \\
Focal cortical dysplasia & $14(35)$ \\
Cryptogenic & $9(22.5)$ \\
Mesial temporal sclerosis & $4(10)$ \\
Tuberous sclerosis & $3(8)$ \\
Other (etiologies with only one & $10(25)$ \\
$\quad$ to two patients) & \\
rs-fMRI score, $N(\%)$ & \\
No abnormality & $2(5)$ \\
Sublobar lesion & $31(77.5)$ \\
Lobe & $5(12.5)$ \\
Hemisphere & $2(5)$ \\
ECoG, $N$ (\%) & $2(5)$ \\
No rs-fMRI-detected abnormality & $38(95)$ \\
Abnormality detected by rs-fMRI & \\
\hline
\end{tabular}

${ }^{\mathrm{a}}$ Other includes one ambidextrous, one neither, and one not yet handed.

CLRE, cryptogenic localization-related epilepsy; ECoG, electrocorticography; rs-fMRI, resting-state functional magnetic resonance imaging; $S D$, standard deviation; SLRE, symptomatic localizationrelated epilepsy. 
fMRI was collected as part of a standard preoperative MRI, no additional consent procedures were necessary. Although specific consent for rs-fMRI sequence was not obtained, all patient families were aware of receiving rs-fMRI. Of the 350 patients, $114(33 \%)$ went on to have surgical resection of seizure foci. The study of rs-fMRI for the first 350 epilepsy patients led to the establishment of a working paradigm to analyze and report the rs-fMRI data in pediatric epilepsy patients, with prior published experience in application of rs-fMRI to correlate with previously established seizure propagation patterns (Boerwinkle et al., 2016).

Evaluation phase. This working paradigm developed was applied to evaluate rs-fMRI data in 40 children with intractable epilepsy who had subsequently undergone ic-EEG and then, if indicated, surgery for seizure control. Analyses focused on the implementation of our working paradigm to analyze rs-fMRI data in pediatric epilepsy, and a comparison to ic-EEG data. Reviewers of the rs-fMRI data were aware that every patient had intractable epilepsy and warranted a surgical evaluation involving operative EEG monitoring, and the ic-EEG results were not known at the time of rs-fMRI analysis. In addition, the practitioners interpreting ic-EEG recordings were not involved in the rs-fMRI analysis. Outcome at 1 year postoperation was determined by using the Engel Classification (Engel 1993).

\section{Image acquisition}

Images were acquired on a 3 Tesla MRI (Ingenuity; Philips Medical Systems, Best, Netherlands) equipped with a 32channel head coil. Thirty-three of 40 patient scans $(83 \%)$ were obtained under light conscious sedation with propofol to reduce patient motion and improve tolerability of the scan. The other seven patients did not need sedation to cooperate. Propofol at levels producing conscious sedation reduces the BOLD signal strength by $\sim 10 \%$, still allowing for complete network detection (Schrouff et al., 2011; Vanderby et al., 2010). General anesthesia causes gross loss of ability to detect the large-scale cortical networks and, was, therefore avoided. Typical scanning parameters were employed. rs-MRI parameters included TR 2,000 msec, TE $30 \mathrm{msec}$, matrix size $80 \times 80$, flip angle $80^{\circ}$, number of slices 46 , slice thickness $3.4 \mathrm{~mm}$ with no gap, in-plane resolution $3 \times 3 \mathrm{~mm}$, and number of total volumes 600 lasting $20 \mathrm{~min}$, well exceeding the accepted minimum required for analysis (Damoiseaux et al., 2006). For anatomic reference, a T1 TFE whole-brain sequence was obtained with TR $9 \mathrm{msec}$, TE $4 \mathrm{msec}$, flip angle $8^{\circ}$, slice thickness $0.9 \mathrm{~mm}$, and in-plane resolution $0.9 \times 0.9 \mathrm{~mm}$. Anatomical images were interpreted by an experienced pediatric neuroradiologist.

\section{Preprocessing}

Analysis was carried out by using a well-known analysis software, the FMRIB Software Library tool MELODIC (Beckmann and Smith, 2004). Standard preprocessing steps were applied: The first five volumes were deleted to remove T1 saturation effects, high pass filter $100 \mathrm{~s}$, inter-leaved slice time correction, spatial smoothing $1 \mathrm{~mm}$, and motion corrected by MCFLIRT (Jenkinson et al., 2002), with non-brain structures removed. All subjects had $<0.5 \mathrm{~mm}$ motion-induced displacement in any direction. This is well under the generally accepted level $(1.0 \mathrm{~mm})$ of subject motion for rs-fMRI investigation. Individual functional scans were registered to the patient's high-resolution anatomical scan by using linear registration (Jenkinson and Smith, 2001), with optimization using boundary-based registration (Greve and Fischl, 2009).

\section{Independent component analysis}

The total number of detected ICs was determined by using the widely established automated dimensionality estimate that utilizes an established Bayesian approach (Beckmann et al., 2005). As such, the threshold of the ICA was set by the standard local false discovery rate for IC detection at $p<0.05$ (Beckmann et al., 2005).

\section{IC categorization}

Working paradigm overview. The working paradigm was developed by a single clinician (V.L.B.) utilizing clinical interpretation and categorization of more than 35,000 ICs from 350 intractable epilepsy patients with the typical patient having an average number of detected ICs of $\sim 100$ (range 35145). Patient ICs were separated into four categories-noise, resting-state network (RSN), EZ, or unknown-by using criteria modified from established norms (Beckmann et al., 2000; Damoiseaux et al., 2006; Pruim et al., 2015; Smith et al., 2009) and novel criteria based on our experience (Fig. 1).

The developed criteria were not designed to be a comprehensive review of all possible considerations or replacement for experienced interpretation but rather a review of the major factors considered for EZ localization. The areas deemed to be the most likely EZ by rs-fMRI did not imply ongoing seizure activity during the rs-fMRI but rather deranged detectable connectivity disruption. The percentage of noise-related ICs was 40-65\%, somewhat less than published norms (Griffanti et al., 2016) and likely related to extremely low patient motion in this population under light conscious sedation. The typical number of retained ICs was 35 and the number of rs-fMRI EZ was between 0 and 6 , with most patients having 2-3.

\section{Noise-IC}

First, ICs were determined to be clearly noise related if they fulfilled any one of the following criteria, similar to previously published work (Griffanti et al., 2017) (Supplementary Fig. S2):

(1) The IC had a spatial pattern that was located:

(a) primarily outside the brain, such as inside the ventricles,

(b) inside major cerebral venous sinuses,

(c) inside major blood vessels,

(d) in the spatial distribution of blood vessels between gyri such as in the operculum, or

(e) most of the IC was located within the white matter.

(2) The IC had a spatial distribution that was consistent with the appearance of MRI-machine-generated artifact in that it was contained within skipping slices of the brain and uniformly distributed over the brain without respect for anatomic boundaries, or in the pattern of a thin even ring at the rim of the brain surface, as such with the effect of head motion; or if the temporal characteristics (i.e., the BOLD time course or frequency power spectrum) clearly fit artifact, such as ultra-slow regular scanner drift time course with frequency less than one-fifth that of normal neuronal oscillation. 


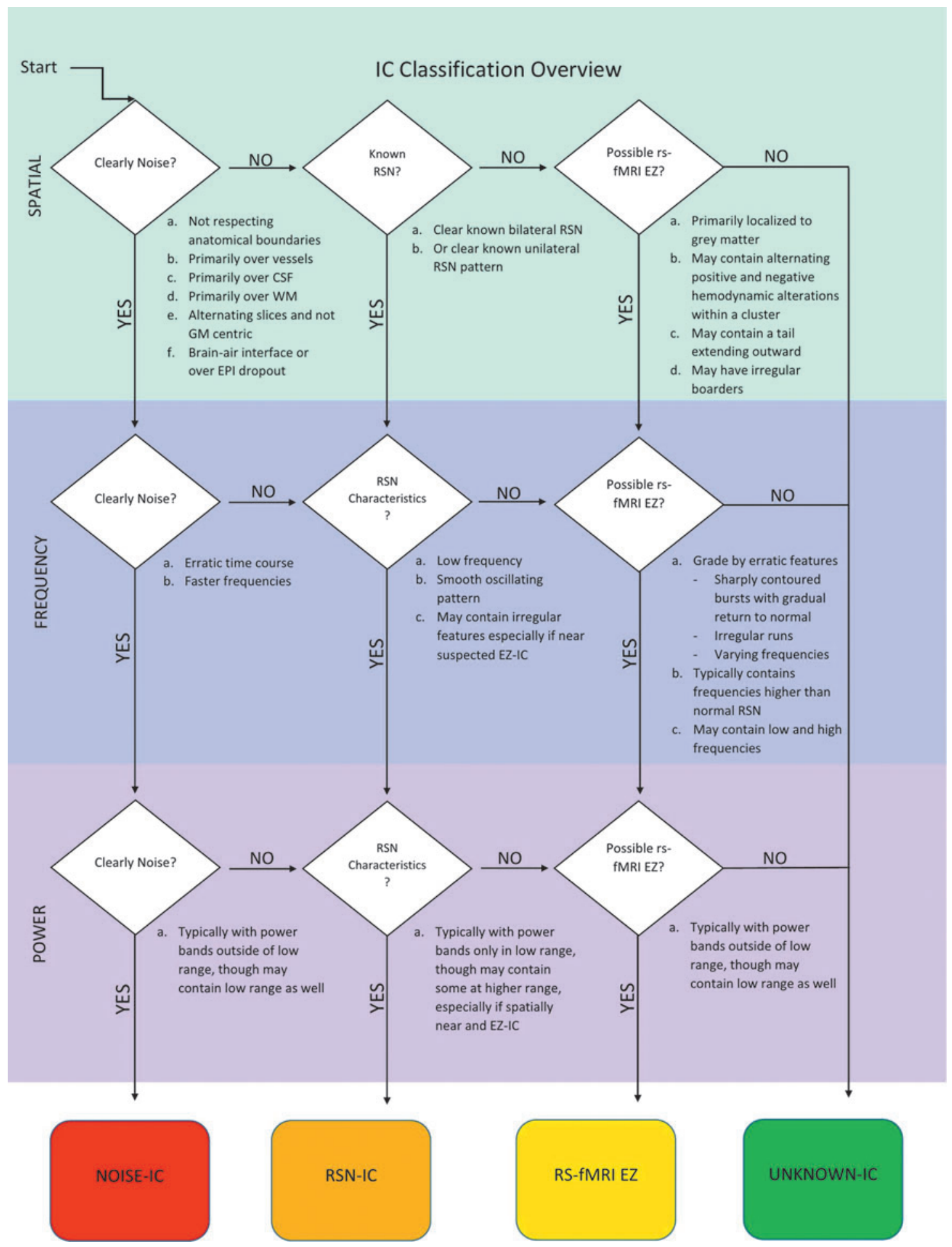

FIG. 1. Independent component classification overview. CSF, cerebral spinal fluid; EPI, resting-state MRI sequence; EZ, epileptogenic zone; GM, grey matter; IC, independent component; RSN, resting-state network; WM, white matter. Color images available online at www.liebertpub.com/brain

(3) A source was considered physiological noise if it met the three criteria of being contained within the frequency range of $0.1-0.5 \mathrm{~Hz}$ for respiratory-related effects or $0.6-1.2 \mathrm{~Hz}$ for cardiac-related effects; having a regular but fast oscillation pattern; and having a spatial pattern within cerebral spinal fluid or vascular structures.

\section{Resting-state network-IC}

(1) ICs not clearly conforming to the noise signal criteria mentioned earlier were sorted visually compared with the spatial features of established RSNs (Supplementary Fig. S1) (Beckmann et al., 2000; Damoiseaux et al., 2006; Smith et al., 2009). 
(2) Those ICs deemed consistent with the RSN spatial pattern were then sorted by established major brainfunction areas-motor, language, parietal, frontal, temporal, occipital, default mode network (a known modulating network), deep gray, long-range association, and other-unique-which aided in the recognition of network temporal feature trends, recognition of missing expected networks, and ability to see what nearby IC features were in relation.

(3) Temporal features of frequency and frequency power spectra were examined and compared with the known low frequency, regular slow oscillating time course, and low-frequency power spectra features of RSN norms (Griffanti et al., 2016).

(4) Temporal features outside of norms were used as contextual features of later deemed rs-fMRI EZ. For example, locally increased RSN temporal atypical features to nearby EZ with a graded decrease in atypical severity as distance increased from the EZ were used as a feature, increasing likelihood that the rsfMRI EZ did disrupt local brain function.

\section{rs-fMRI epileptogenic zone}

(1) The IC had a spatial pattern not conforming to published norms for noise or RSN (Supplementary Fig. S3) (Beckmann et al., 2000; Damoiseaux et al., 2006; Griffanti et al., 2016; Hunyadi et al., 2015; Pruim et al., 2015; Smith et al., 2009), such as:

(a) being more asymmetrically unilateral than expected for the IC in that area, or

(b) with an alternating localized activation-deactivation pattern over gray matter, or

(c) with a tapered tail from the cortex extending toward the ventricle.

(2) The IC had an irregular time course, in comparison to the more regular time courses of the other RSN-ICs (see case examples for appearance and further descriptions of such irregular time courses).

(3) The IC contained frequency $>0.4 \mathrm{~Hz}$ (see case-based frequency graphs for examples), and in the context of the individual's other neuronal IC characteristics was relatively either spatially or temporally more atypical (Beckmann et al., 2000).*

*Note that in studies of normal individuals, this criterion is not used and the frequency cutoff between normal neuronal network activity is generally considered between 0.01 and $0.1 \mathrm{~Hz}$ and physiological noise is above $0.4 \mathrm{~Hz}$ (Biswal et al., 1995). Historically, this cutoff point between normal neuronal signal and physiological ICs was used to avoid corruption of functional connectivity measures with physiological noise. This was necessary because without such severe filtering, the traditional functional connectivity measure used, the correlation coefficients between signal level between brain voxels, would be corrupted with too much signal from physiological noise. In contrast, since the functional connectivity measure from ICA does not use gross signal level in a correlation measure but rather, the ICA segregates ICs based on maximizing the difference between Gaussian versus non-Gaussian signal features, it is not necessary to filter at the traditional physiological noise cutoff and may possibly reduce localization of tissue most affected by the pathology of the epilepsy (Cordes et al., 2001). In this study, only ultra-slow frequencies $(<0.01 \mathrm{~Hz})$, below typical neuronal ICs, were filtered, allowing faster frequency information to remain.

\section{Unknown-IC}

(1) IC not clearly meeting any of the categories mentioned earlier or possibly containing mixed features of more than one category were reported separately.

(2) Unknown-IC themselves counted as a feature that contributed to the nearby rs-fMRI EZ as a local feature, increasing the likelihood that rs-fMRI EZ disrupted local brain function.

\section{rs-fMRI EZ ranking and contextual interpretation}

Those ICs deemed clearly to not be noise or RSN related were further considered:

(1) Spatial rs-fMRI EZ features from least atypical to most atypical

(a) Located in exactly the same area as known RSN but incomplete spatial coverage (these IC were almost uniformly deemed downstream epileptogenic propagation phenomena as opposed to rsfMRI EZ).

(b) Contours of clusters with increased irregularity to borders (note data smoothing not performed).

(c) Located anatomically outside of RSN spatial norms and primarily in gray matter.

(d) Located in gray matter with tail extending into white matter.

(e) Located primarily in gray matter with alternating negative and positive BOLD IC signal values.

(2) Frequency and time course rs-fMRI EZ features from least atypical to most atypical

(a) The IC has a time course that is a clear, smoothly sinusoidal oscillation pattern, which is more consistent with a normally functioning neuronal network.

(b) The IC has a time course that is still a regular sinusoidal oscillation pattern but is overlaid with either regular or irregular faster frequency, which is consistent with a neuronal network with atypical frequency characteristics compared with normal.

(c) The IC has a time course that is a regular sinusoidal pattern interrupted by sharply contoured bursts of activity with a gradual return to normal. If the subject has no other component consistent with major disruption at the same time point, such as a patient motion component, then this may be consistent with the nearby effect of epilepsy.

(d) The IC has a time course that contains runs of irregular and sharply contoured oscillations that vary in frequency, with faster and more erratic patterns than the other neuronal appearing ICs. (See the Discussion section for comparison of these criteria with previously proposed temporal characteristic classification schemes.)

(3) Frequency power spectra features from least atypical to most atypical

(a) Frequency power band primarily confined to $<0.039 \mathrm{~Hz}$.

(b) Power band containing increasing amounts of frequency higher than $0.039 \mathrm{~Hz}$ but not 
uniformly confined to the narrow cardiac and respiratory bands.

(c) Increasing number of clusters of power bands included.

\section{Other rs-fMRI EZ contextual grading features}

Other types of atypical differences were noted but were given substantially less weight than the features cited earlier. These included missing networks that were expected to be detected given the child's overall reported skill level, or networks detected in the deactivated state, though this finding has no known significance and was given the least weight of all. These features were considered soft signs of dysfunction in that given area but in and of themselves were not generally considered as an EZ.

\section{Further contextual rs-fMRI EZ grading}

If more than one abnormal IC existed, they were compared with one another and ranked in degree of overall abnormality of their spatial and temporal features. In many cases, the abnormal ICs clustered on the same area of gray matter, and together were deemed the most abnormal area and most likely to be the source of seizure. If two equally abnormal ICs were identified in spatially separate locations, the study was declared to have more than one EZ. If many (three or greater) spatially separate and equally abnormal areas were identified, the study was deemed to be consistent with multifocal or possibly generalized epilepsy. If such differentiation could not be clearly made, the study was deemed nonlocalizing by rsfMRI. (See the Discussion section for details on subjectivity.)

\section{ic-EEG ictal source localization}

Patients underwent workup and identification as epilepsy surgery candidates based on recommendation from a multidisciplinary epilepsy surgery program. Seizure zone or zones were targeted based on hypotheses generated from a combination of anatomic imaging, functional and metabolic imaging (PET, SPECT), prolonged video-monitored electroencephalogram, brain electrical source analysis, and/or magnetoencephalogram. Surgical targets were identified by coregistration of the EZ with lobar or gyral anatomy on MRI. Stereotactic planning software and intraoperative neuronavigation systems (BrainLab, iPlan, BrainLab Curve) were uti- lized for both invasive monitoring and intraoperative monitoring. Under sterile conditions and epilepsy surgery anesthetic protocols of total intravenous anesthesia, patients underwent craniotomy or burr holes for placement of invasive monitoring electrodes, utilizing subdural electrodes or depth electrodes as indicated. For this article, we use the term icEEG inclusively to denote electrocorticographic intraoperative monitoring that may be extended extraoperatively. Table 2 details the breakdown in the monitoring technique used and the localization based on ictal and interictal icEEG data gathered. The placement of the recording electrodes was correlated with anatomic photographs and by threedimensional imaging with computed tomography fused with preoperative MR images. Both Ad-Tech Medical Instrument Corporation (Racine, WI) and Integra Life Sciences (Plainsboro, NJ) surface and depth electrodes were used in this study.

Identification of SOZs from invasive electrode data was based on expert review and interpretation by experienced pediatric epileptogists of the pediatric surgical epilepsy program. Plans for surgical resection were developed based on these findings with consensus from the epilepsy and neurosurgical teams.

\section{Scoring of rs-fMRI and ic-EEG and surgical resection cavity}

Co-registration of rs-fMRI-determined EZ to lobar or gyral anatomy on anatomical MRI allowed for visualizing overlap of gray matter with the area studied by ic-EEG, and this yielded consistent spatial correspondence to the ic-EEGdetermined SOZ. ic-EEG was scored as "yes" or "no" for seizure activity in the location of the individual electrodes by an expert pediatric epileptologist. The location(s) of the EZ by rsfMRI was scored by two raters independently (D.M. and V.L.B.) in relation to the location of the ic-EEG electrodes as one of the following: (1) no seizure activity, (2) EZ localized to the exact same location of the ic-EEG sensors detected SOZ, (3) seizure activity in the same entire lobe, and (4) generalized seizure activity in the whole same hemisphere.

Determination of degree of ic-EEG SOZ and rs-fMRI EZ overlap. The correspondence of anatomical boundaries of the ic-EEG SOZ and rs-fMRI EZ was determined by two independent expert reviewers (D.M. and V.L.B.) via visual review of the co-registered images with the following grading scale:

Table 2. Operative (Electrocorticography) Versus Extra-Operative (Stereo-Electroencephalogram and Grid Placement) Monitoring Agreement

\begin{tabular}{|c|c|c|c|c|c|}
\hline \multirow[b]{2}{*}{ Seizure } & \multicolumn{2}{|c|}{ Operation } & \multirow[b]{2}{*}{ Total } & \multirow[b]{2}{*}{ Agreement with rs-fMRI } & \multirow[b]{2}{*}{ Ordinal scale PABAK } \\
\hline & Operative & Extra-operative & & & \\
\hline Ictal & 3 & 15 & $18(45 \%)$ & $94 \%(73 \%, 100 \%)$ & $0.889(0.679,1)$ \\
\hline Interictal & 20 & 2 & $22(55 \%)$ & $86 \%(65 \%, 97 \%)$ & $0.727(0.442,1)$ \\
\hline Total & 23 & 17 & 40 & & \\
\hline
\end{tabular}

Operative \%: Ictal 13\%; extra-operative \%: Ictal $88 \% ; p<0.001$ (Fisher's exact test).

Forty-five percent of subjects had ictal studies, and 55\% only had interictal activity. Of those with ictal studies, agreement with rs-fMRI-based localization was $73 \%$ for the operative ECoG group versus $100 \%$ with the extra-operative studies. Similarly, of those with only interictal studies, agreement with rs-fMRI-based localization, $65 \%$ for the operative-based ECoG group and $97 \%$ agreement in the extra-operative group, averaging as cited earlier. No statistical difference was detected in the agreement rate with rs-fMRI based on the EEG monitoring method.

EEG, electroencephalogram; PABAK, prevalence-adjusted bias adjusted kappa. 
Table 3. Electrocorticography and Resting-State Functional Magnetic Resonance Imaging Level of Spatial (Ordinal) Agreement

\begin{tabular}{|c|c|c|c|c|c|}
\hline \multirow[b]{2}{*}{$E C o G$} & \multicolumn{4}{|c|}{$r s-f M R I$} & \multirow[b]{2}{*}{ Total } \\
\hline & No abnormality & Lesion/part of the lobe & Lobe & Hemisphere & \\
\hline No abnormality & 0 & 0 & 1 & 1 & 2 \\
\hline Abnormality & 2 & 31 & 4 & 1 & 38 \\
\hline Total & 2 & 31 & 5 & 2 & 40 \\
\hline
\end{tabular}

(1) No difference: Anatomical boundaries are visually the same.

(2) Sub-lobar partial overlap: The majority $(>50 \%)$ of the ic-EEG SOZ and rs-fMRI EZ overlap and are contained within a boundary less than one lobe.

(3) Lobar partial overlap: The majority of the ic-EEG SOZ and rs-fMRI EZ overlap and are contained within a boundary of one lobe.

(4) Hemispheric partial overlap: The majority of the icEEG SOZ and rs-fMRI EZ overlap and are contained within a boundary of one hemisphere.

(5) Nonoverlapping regions were not considered in agreement between rs-fMRI and ic-EEG.

When disagreement occurred, the raters reviewed the data together and consensus was achieved in the few cases of initial discrepancy.

Determination of degree of ic-EEG SOZ, rs-fMRI EZ, and surgical resection/disconnection overlap. Further analysis was performed to correlate the extent of surgical resection, defined as the area of brain actually resected, ablated by laser, or functionally disconnected and the anatomical boundaries of the ic-EEG SOZ and rs-fMRI EZ pre- and postoperatively as determined by the two independent expert reviewers (D.M. and V.L.B.) via visual review of the images.

\section{Outcome}

To determine outcome for the group of surgical patients, seizure freedom percentages (either seizure free or $>50 \%$ seizure reduction) were collected at 6 and 12 months after surgery. Further, each patient was classified by using the Engel Classification and the categorization was modified to reflect 1 year postoperative seizure classification. Definitions of distribution of patient outcomes were determined from clinical data by pediatric neurologists. Given the relatively broad range of outcomes of children with Engle class III status, each of these patients was individually reviewed to determine categorization of being a true or false positive as described in Table 8 .

\section{Statistical analysis}

When examining agreement between the ic-EEG and rsfMRI findings, the rs-fMRI score was assessed as dichotomous (no seizure activity vs. seizure activity overlapping with ic-EEG electrodes but not necessarily localized to the exact same anatomical boundaries) as well as level of spatial agreement, as seen in Table 3 . The proportion of agreement between methods with $95 \%$ binomial exact confidence interval (CI) was calculated for both dichotomous and ordinal rs-fMRI. The agreement of ic-EEG SOZ and dichotomous rs-fMRI EZ was assessed by using the prevalence-adjusted bias adjusted kappa (PABAK) (Byrt et al., 1993). Agreement between ic-EEG SOZ and ordinal rs-fMRI EZ localization was similarly assessed by using PABAK for ordinal scale (PABAK-OS). The PABAK values were interpreted as follows: $\leq 0$ poor agreement, $0.01-$ 0.20 slight agreement, $0.21-0.40$ fair agreement, $0.41-0.60$ moderate agreement, $0.61-0.80$ substantial agreement, and 0.81-1.0 almost perfect agreement (Landis and Koch, 1977). Since the kappa statistic may be affected by the high prevalence of seizure activity in this patient population, PABAK (Byrt et al., 1993) was used to assess agreement instead of the traditional kappa statistic (Stata Corp, College Station, TX; single case research; Web-based calculators for SCR analysis. 1.0) as seen in Table 2. Differences in seizure outcome were checked for correlation with the testing condition of sedation during the rs-fMRI versus not sedated. The Fisher's exact test examined the association between rs-fMRI EZ and Engel Classification outcome at 1 year. Sensitivity and positive predicted values were estimated with corresponding 95\% CIs. All statistical tests were two sided, with significance evaluated at the 5\% level. Analyses were conducted by using Stata version 12.1 .

\section{Results}

Patient demographics and clinical characteristics are summarized in Table 1 . The mean age was 12 years, and more than half of the patients were men. More than three-quarters of patients had symptomatic localization-related epilepsy (SLRE). Abnormal anatomical imaging findings, the most common etiology being focal cortical dysplasia (35\%), were present in $95 \%$ of patients included in this study with preoperative rs-fMRI done before ic-EEG.

The observed agreement between ic-EEG SOZ and dichotomous rs-fMRI EZ (Table 4) was 90\% ( $N=36 / 40$; 95\% CI, 0.76-0.97). The PABAK estimate was $0.80(95 \%$ CI, 0.61-0.99), indicating substantial agreement between the two methods. Similarly, the observed agreement between ic-EEG SOZ and ordinal rs-fMRI EZ (Table 3) was $78 \%$

Table 4. Electrocorticography and Resting-State Functional Magnetic Resonance Imaging (Dichotomous) AgreEMENT

\begin{tabular}{lccc}
\hline & \multicolumn{3}{c}{$r s-f M R I$} \\
\cline { 2 - 3 } ECoG & No abnormality & Abnormality & Total \\
\hline No abnormality & 0 & 2 & 2 \\
Abnormality & 2 & 36 & 38 \\
Total & 2 & 38 & 40
\end{tabular}


$(N=31 / 40 ; 95 \%$ CI, $0.62-0.89)$ with a PABAK value of 0.70 (95\% CI, 0.57-0.83). Overall, observed agreement was high, and the PABAK values indicated substantial to a high level of agreement for the dichotomous rs-fMRI EZ and the ordinal rs-fMRI EZ.

Agreement in those with cryptogenic location-related epilepsy (CLRE) was 89\% (8/9; 95\% CI, 0.52-99), with no statistical difference between the agreement in SLRE and CLRE subgroups.

There were four instances of disagreement between the two methods: two cases with negative rs-fMRI EZ, who had the ic$\mathrm{SOZ}$ area resected and were seizure free; and two children who had negative ic-EEG and positive rs-fMRI EZ. In this latter scenario, the two children in whom intraoperative ic-EEG monitoring did not detect seizure activity at the rs-fMRI location went on to have surgical removal of the rs-MRI lesion based on and supported by total collected evidence of all modalities of testing done. Surgical planning was not made on the basis of rs-fMRI EZ localization but rather the consensus of the concordance of all testing and the collective judgment of the epilepsy surgery team. These two patients remained seizure free 1 year after surgery and, therefore, were considered true positives for EZ. The two raters consistently identified agreement between rs-fMRI EZ and ic-EEG SOZ findings for 34 ( $85 \%$ of 40 ) subjects. For the six subjects in whom some un- certainty in rs-fMRI EZ and ic-EEG SOZ agreement was noted by either rater, consensus was reached after joint review by the raters. Further, raters were consistent in classifying postoperative surgical imaging and rs-fMRI SOZ for all subjects.

As shown in Table 2, there was no statistical difference between the degrees of agreement between rs-fMRI EZ and icEEG SOZ localization when sub-grouped by ic-EEG findings of ictal versus interictal ( $p=0.613$; Fisher's exact test). For patients who received intraoperative monitoring, agreement was $87 \%$ (95\% CI, 66-97\%) and PABAK $=0.74$ (95\% CI, 0.4661); for those who received extraoperative monitoring, agreement was 94\% (95\% CI, 71-100\%) and PABAK $=0.883(95 \% \mathrm{CI}$, 0.661-1) with no significant difference with respect to the rsfMRI localization of EZ ( $p=0.624$; Fisher's exact test).

An overlap of multiple abnormal ICs occurred in 19 subjects, whereas 18 subjects had only one IC declared as the most abnormal when compared with the remainder of the patient's other ICs, and there was no statistical difference in the 1-year seizure freedom rate between these two groups. Two patients did not have an abnormal rs-fMRI EZ IC. One patient had an atypical finding, which was not declared an EZ but was still considered the subject's most abnormal IC.

Table 5 shows that both CLRE and SLRE display high agreement rates of ic-EEG SOZ with rs-fMRI EZ. Among those with SLRE, the subgroup with focal cortical dysplasia

Table 5. Agreement Between Electrocorticography and Resting-State Functional Magnetic Resonance Imaging Sub-Classified by Epilepsy Type and Cause

\begin{tabular}{|c|c|c|c|c|c|c|c|c|}
\hline & $E C o G, \mathrm{~N}$ & & $\begin{array}{l}\mathrm{N}, \text { rs-fMRI } \\
\text { agree with } \\
\text { ECoG, \% } \\
(95 \% \text { CI })\end{array}$ & $\begin{array}{l}P A B A K \\
(95 \% C I)\end{array}$ & Surgery, & $\begin{array}{l}\text { Seizure-free } \\
1 \text { year, } \\
\mathrm{N}(\%)\end{array}$ & $\begin{array}{l}\text { Seizure-free } \\
6 \text { months, } \\
\mathrm{N}(\%)\end{array}$ & $\begin{array}{c}\text { With }>50 \% \\
\text { seizure } \\
\text { reduction } \\
6 \text { months, N }\end{array}$ \\
\hline Overall & 40 & 36 & $90(76-97)$ & $0.80(0.61-0.99)$ & 37 & $20(54)$ & $24(65)$ & $31(84)$ \\
\hline $\begin{array}{l}\text { Seizure classification } \\
\text { SLRE } \\
\text { CLRE }\end{array}$ & $\begin{array}{r}31 \\
9\end{array}$ & $\begin{array}{r}28 \\
8\end{array}$ & $\begin{array}{l}90(74-98) \\
89(52-99)\end{array}$ & $\begin{array}{l}0.81(0.60-1) \\
0.60(0.10-1)\end{array}$ & $\begin{array}{r}30 \\
7\end{array}$ & $\begin{array}{r}18(60) \\
2(29)\end{array}$ & $\begin{array}{r}22(73) \\
2(29)\end{array}$ & $\begin{aligned} 27 & (90) \\
4 & (57)\end{aligned}$ \\
\hline $\begin{array}{l}\text { Fisher's exact test } \\
\quad p \text {-value }\end{array}$ & & & 0.999 & & & 0.212 & 0.079 & 0.068 \\
\hline \multicolumn{9}{|c|}{ Primary etiology (SLRE only) } \\
\hline $\begin{array}{l}\text { Focal cortical } \\
\text { dysplasia }\end{array}$ & 14 & 12 & $86(57-98)$ & $0.71(0.35-1)$ & 13 & $7(54)$ & $9(69)$ & $11(85)$ \\
\hline $\begin{array}{l}\text { Mesial temporal } \\
\text { sclerosis }\end{array}$ & 4 & 4 & $100(40-100)$ & $\mathrm{n} / \mathrm{a}$ & 4 & $3(75)$ & $3(75)$ & $4(100)$ \\
\hline CNS tumor & 6 & 5 & $83(39-99)$ & $\mathrm{n} / \mathrm{a}$ & 6 & $4(67)$ & $5(83)$ & $6(100)$ \\
\hline Tuberous sclerosis & 3 & 3 & $100(29-100)$ & $\mathrm{n} / \mathrm{a}$ & 3 & $2(67)$ & $2(67)$ & $2(67)$ \\
\hline Encephalitis & 2 & 2 & $100(16-100)$ & $\mathrm{n} / \mathrm{a}$ & 2 & $1(50)$ & $1(50)$ & $2(100)$ \\
\hline $\begin{array}{l}\text { Congenital } \\
\text { malformation }\end{array}$ & 2 & 2 & $100(16-100)$ & $\mathrm{n} / \mathrm{a}$ & 2 & $1(50)$ & $2(100)$ & $2(100)$ \\
\hline $\begin{array}{l}\text { Fisher's exact test } \\
p \text {-value }\end{array}$ & & & 0.999 & & & 0.984 & 0.978 & 0.661 \\
\hline \multicolumn{9}{|c|}{ rs-fMRI score-type of localization of focus } \\
\hline (1) Sublobar & 31 & 31 & $100(89-100)$ & $0.82(0.72-1)$ & 28 & $14(50)$ & $18(64)$ & $24(86)$ \\
\hline (2) Lobar & 5 & 4 & $80(28-99)$ & $\mathrm{n} / \mathrm{a}$ & 5 & $4(80)$ & $4(80)$ & $4(80)$ \\
\hline (3) Hemispheric & 2 & 1 & $50(1-99)$ & $\mathrm{n} / \mathrm{a}$ & 2 & $1(50)$ & $1(50)$ & $2(100)$ \\
\hline $\begin{array}{l}\text { (4) No epileptiform } \\
\text { anomaly }\end{array}$ & 2 & 0 & $0(0)$ & $\mathrm{n} / \mathrm{a}$ & 2 & $1(50)$ & $1(50)$ & $1(50)$ \\
\hline $\begin{array}{l}\text { Fisher's exact test } \\
p \text {-value }\end{array}$ & & & 0.001 & & & 0.829 & 0.922 & 0.627 \\
\hline
\end{tabular}

Seizure freedom outcome at 1 year, and also 6 months; percent with $>50 \%$ reduction in seizure frequency. CI, confidence interval; CNS, central nervous system. 
was powered to determine the agreement between rs-MRI EZ and ic-EEG SOZ. Seizure freedom percentages are reported for 6 and 12 months after surgery (65\% and 54\%, respectively). Overall, $84 \%$ and $65 \%$ of patients had at least a $50 \%$ reduction in seizures 6 and 12 months after surgery, respectively. rs-fMRI with sedation versus without sedation did not have a statistically significant effect on the outcome of seizure freedom $(p=0.407)$.

Notably, there were four patients for whom the rs-fMRI EZ did not agree with the location of the intraoperative monitoring. Two of these patients did not have a detectable rs-fMRI EZ and had Engel 1 and 3 outcomes. The other two patients had rs-fMRI EZs that were surgically destroyed despite the lack of intraoperative monitoring agreement and were Engel Class 1 at 1 year postoperative outcome. There were no patients who lacked ic-EEG and rs-fMRI EZ agreement and had rs-fMRI EZ remaining after surgery.

A significant association (Fisher exact $p=0.01$ ) was detected between resection of the rs-fMRI EZ and Engel categorization of seizure activity at 1 year (Table 6). As shown in Tables 6 and 7, 8 (24\%) out of the 33 patients had some agreement between rs-fMRI EZ and ic-EEG SOZ but had rs-fMRI EZs anatomically separate that remained after the surgical resection (i.e.) part of rs-fMRI EZ was not removed. Of these patients, six (75\%) patients continued to have seizures 1 year later. Conversely, among 25 patients who had some agreement between rs-fMRI EZ and ic-EEG SOZ and had no anatomically separate rs-fMRI EZ clusters after resection, only $6(24 \%)$ continued to have seizures 1 year later. The presence of un-resected rs-fMRI EZ clusters was significantly correlated to continued seizures after surgery (Fisher's exact test; $p=0.01$ ).

Summarizing the results from Tables 6 and 7, half of the cases with sub-optimal outcomes (Engel 2-4), that is 6/12, had rs-fMRI-positive clusters that were not surgically destroyed. On the other hand, of the 21 with good outcomes (Engel 1), only 2 cases had rs-fMRI-positive clusters that did not overlap with the resection area. Of the 37 patients who had surgical destruction of the area believed to be the primary source of seizures, 27 (73\%) rs-fMRI EZ could be classified as true positives, 7 (19\%) false positives, and $2(5 \%)$

Table 6. Distribution of Patients According to Resting-State Functional Magnetic Resonance IMAGING EPILEPTOGENIC ZONE AND ENGEL CLASSIFICATION

\begin{tabular}{|c|c|c|c|c|c|}
\hline \multirow[b]{2}{*}{$\begin{array}{l}r s-f M R I E Z, \\
\mathrm{~N}(\%)\end{array}$} & \multicolumn{4}{|c|}{ Engel } & \multirow[b]{2}{*}{$\mathrm{p}^{\mathrm{a}}$} \\
\hline & $\begin{array}{c}1 \\
(\mathrm{~N}=21)\end{array}$ & $\begin{array}{c}2 \\
(\mathrm{~N}=1)\end{array}$ & $\begin{array}{c}3 \\
(\mathrm{~N}=7)\end{array}$ & $\begin{array}{c}4 \\
(\mathrm{~N}=4)\end{array}$ & \\
\hline $\begin{array}{l}\text { Resected }^{\mathrm{b}} \\
\text { Not resected }\end{array}$ & $\begin{array}{r}19(90) \\
2(10)\end{array}$ & $\begin{array}{l}0(0) \\
1(100)\end{array}$ & $\begin{array}{l}3(43) \\
4(57)\end{array}$ & $\begin{array}{l}3(75) \\
1(25)\end{array}$ & 0.01 \\
\hline
\end{tabular}

Resection refers to surgical destruction, including laser ablation, resection, and functional disconnection. All patients in this table had the ic-EEG SOZ resected.

${ }^{a} p$-Value from Fischer's exact test.

${ }^{b}$ rs-fMRI EZ anatomically agreed with the ic-EEG SOZ and was resected.

${ }^{c}$ rs-fMRI EZ was anatomically separate from the ic-EEG and was not resected.

EZ, epileptogenic zone; ic-EEG, intracranial electroencephalogram; SOZ, seizure onset zone.
Table 7. Distribution of Patients AcCording

to Resection of Resting-State Functional

Magnetic Resonance Imaging EPILEPTOGENIC

Zone and Engel Classification (2-4 Combined)

\begin{tabular}{|c|c|c|c|}
\hline \multirow[b]{2}{*}{$r s-f M R I E Z, \mathrm{~N}(\%)$} & \multicolumn{2}{|c|}{ Engel } & \multirow[b]{2}{*}{$\mathrm{p}^{\mathrm{a}}$} \\
\hline & $1(\mathrm{~N}=21)$ & $2-4(\mathrm{~N}=12)$ & \\
\hline Resected $^{\mathrm{b}}$ & $19(90)$ & $6(50)$ & 0.02 \\
\hline Not resected $^{\mathrm{c}}$ & 2 (10) & $6(50)$ & \\
\hline
\end{tabular}

Resection refers to surgical destruction, including laser ablation, resection, and functional disconnection.

${ }^{a} p$-Value from Fischer's exact test.

brs-fMRI EZ anatomically agreed with the ECoG SOZ and was resected.

${ }^{c}$ rs-fMRI EZ was not resected.

false negatives (Table 8). Sensitivity of rs-fMRI EZ was $93 \%(95 \% \mathrm{CI}, 78-98 \%)$ with a positive predictive value of $79 \%$ (95\% CI, 63-89\%).

\section{Case Discussion Overview}

The most typical localizing results are similar to those described in Case 1 (Fig. 2) who had several abnormal ICs centered in the same area. There was a gradation of atypical features as the distance increased from the abnormal area. Other times, only one abnormal IC was detected without further surrounding abnormalities to provide context, as exemplified in Case 2 (Fig. 3). Case 3 (Fig. 4) demonstrates the subtle nuances involved in ICA interpretation. The most abnormal IC in this case was based primarily on the unusual spatial features, especially in the right anterior temporal lobe (seen in red). If this feature had not been present, then at best, the interpretation would have still localized the abnormality to the frontotemporal area. Case 4 (Fig. 5) is representative of situations in which rs-fMRI detects nonoverlapping, abnormal ICs, suggesting multifocal or two seizure generators causing epilepsy. In this case, the patient was believed to have more than one seizure source by multimodality testing, though only one region was studied by ic-EEG and removed. This was a palliative decision, with the goal of removing the most active seizure source. Hence, in instances such as these, the interpretation of the ic-EEG and rs-fMRI was determined to be concordant, though there was a spatially separate rs-fMRI abnormal IC.

\section{Discussion}

\section{Salient findings}

This is the largest study to date examining functional connectivity EZ localization by rs-fMRI in epilepsy, and we report a high level of agreement (90\%) between rs-fMRI EZ with ic-EEG SOZ localization. Uniquely, we developed a clinically applicable working paradigm for identifying and grading rs-fMRI EZ, in the context of surrounding artifact-IC, RSN-IC, and unknown-IC's spatial and temporal features that is critical in the overall context of rs-fMRI EZ interpretation. The algorithm was applied to a heterogeneous pediatric epilepsy population, including those with multifocal epilepsy and CLRE. Arguably, this study's population provided the most challenging level of heterogeneity to investigate this 
Table 8. Patient Distribution and Description of Defined Resting-State Functional Magnetic Resonance Imaging Epileptogenic Zone “Truth” in Predicting Actual Seizure Freedom Postoperatively

\begin{tabular}{|c|c|c|}
\hline \multirow{2}{*}{$\begin{array}{l}\text { Predicted } \\
\text { condition }\end{array}$} & \multicolumn{2}{|c|}{ True condition } \\
\hline & Positive & Negative \\
\hline \multirow[t]{7}{*}{ Positive } & $N=27$ & $N=7$ \\
\hline & True positives & False positives \\
\hline & rs-fMRI EZ resected, outcome Engel $1(n=21)$ & All rs-fMRI EZs resected, agreed with ic-EEG, \\
\hline & & but outcome $<50 \%$ seizure reduction and Engel \\
\hline & rs-fMRI EZ resected, and agreed with ic-EEG & $3,4(n=3)$ \\
\hline & SOZ, outcome Engel 2-3 $(n=3)$ & or \\
\hline & $\begin{array}{c}\text { or } \\
\text { rs-fMRI EZ anatomically separate from ic-EEG, } \\
\text { and rs-fMRI EZ not resected, outcome } \\
\text { Engel 3-4 }(n=3)\end{array}$ & $\begin{array}{c}\text { rs-fMRI EZ not resected, did not agree with ic- } \\
\text { EEG, outcome Engel } \\
1-3(n=4)\end{array}$ \\
\hline \multirow[t]{3}{*}{ Negative } & $N=2$ & $N=0$ \\
\hline & False negatives & True negatives \\
\hline & $\begin{array}{l}\text { rs-fMRI EZ not detected but ic-EEG detected } \\
\text { SOZ, outcome Engel } 1-3\end{array}$ & $\begin{array}{l}\text { Neither rs-fMRI EZ nor ic-EEG SOZ } \\
\text { detected, outcome Engel 3-4 }\end{array}$ \\
\hline
\end{tabular}

Sensitivity $93 \%$ (95\% CI 78-98\%). Positive predictive value 79\% (95\% CI 63-89\%). The three children with rs-fMRI EZ and ic-EEG SOZ agreement and resection were considered true positives because the seizure reduction was $>90 \%$, with comparatively extremely minimal seizures postoperatively with similar semiology, pointing toward residual tissue from a prior source as the same SOZ driver most likely. In the four patients with a nonresected rs-fMRI separate from the resected ic-EEG SOZ, one was indeterminate, another was a clear false positive, and two were considered true positives. Of these two patients considered true positives, both had long-standing dual seizure semiologies, one of which continued postoperatively, and the other resolved, indicating a separate seizure source clinically.

rs-fMRI-based EZ localization paradigm but allowed for testing of this method in real-world evaluation and management of children with medically intractable epilepsy. The uniqueness of our analysis is that it is a data-driven approach that generates ICs and can reliably clinically identify rs-fMRI EZs without the requirement of EEG data.

This study found a high degree of agreement between rsfMRI EZ and ic-EEG in both symptomatic (90\%) and cryptogenic $(89 \%)$ localization epilepsy, with no statistical difference between these groups. Second, the agreement between rs-fMRI EZ and ic-EEG SOZ was not dependent on the mode of the surgical monitoring approach (depths vs. surface) nor on whether the recorded cortical activity was ictal versus interictal (Table 2). Localization-based categorization showed that the sub-lobar group had $100 \%$ agreement. Other subgroups, such as lobar and hemispheric, had less agreement, though this was limited by the small sample size. These data would support the fact that rs-fMRI EZ

FIG. 2. Case 1: (A-D) are noted inside the figure on the left side in alphabetical order, each referring to the graphs located just above the letter. Each detected IC from rs-fMRI has two associated temporal or frequency characteristic graphs. The redcolored graph is the BOLD signal level plotted versus time, known as the time course. Normal for this signal is smooth and slowly oscillating. The blue graph is the power spectrum of frequencies detected for that particular IC. Normal for this spectrum is very low, less than the first bar shown on the $x$ axis of the plot. The top set of images are the rs-fMRI abnormal ICs in a patient with extensive chronic right hemispheric encephalomalacia. Each abnormal IC is displayed in a different color. The highest concentrated area of overlap in these ICs was over primary lateral right sensory motor cortex, the same area identified by ic-EEG. Note that there were five abnormal ICs clustered here, each shown in a different color, with one of the IC temporal characteristic graphs shown below as representative of the five. The top red-colored graph is the network time course as signal versus time in seconds, and the bottom blue-colored graph is the power spectrum in power versus frequency in Hz/100. The most atypical portions of the time course are denoted by a black box. (A) Temporal characteristic graphs of the abnormal right frontal lateral M1-S1 network shown in green above. By comparing the normal smooth time course in (D), the spike-like appearance of the atypical ICs in (A-C) may be recognized. Also, the blue graph shows that the power spectrum of the frequencies from this IC is beyond the normal limit of $3.39 \mathrm{~Hz} / 100$. (B) Mesial temporal area frequency-based characteristic graphs, shown for comparison, demonstrate a similar appearing time course as frontal, though slightly less fast, and power spectra over $3.39 \mathrm{~Hz} / 100$. This demonstrates the invasion of surrounding networks with corrupted connectivity to a lesser degree than those closer in spatial proximity to the proposed EZ. (C) Shown for comparison is this patient's "typical" appearing bilateral network within the superior temporal gyri, with a somewhat faster time course than totally normal but more regular in oscillation pattern than those described in $(\mathbf{A}, \mathbf{B})$ with a decreased degree of power spectra spread into higher frequencies. The bottom MRI images are postoperative, demonstrating the effects of the occipital-sparing partial-functional hemispherectomy, and the patient is now seizure free 2 years postoperatively. (D) Postoperative MRI anatomical images define the ic-EEG SOZ, to compare with the preoperative rs-fMRI EZ, specifically available only in this patient. Postoperative images demonstrate the normalization of IC frequency characteristics. This source is located in the same left superior temporal gyrus area as IC shown in (C) above. Note that postoperative rs-fMRI was not uniformly applied and is not an end-point of the study, though it is presented here as a contrast for normal in the same patient. See the Future Directions section for more details in this regard. BOLD, blood-oxygen-level-dependent; ic-EEG, intracranial electroencephalogram; rs-fMRI, resting-state functional magnetic resonance imaging; SOZ, seizure onset zone. Color images available online at www.liebertpub.com/brain 

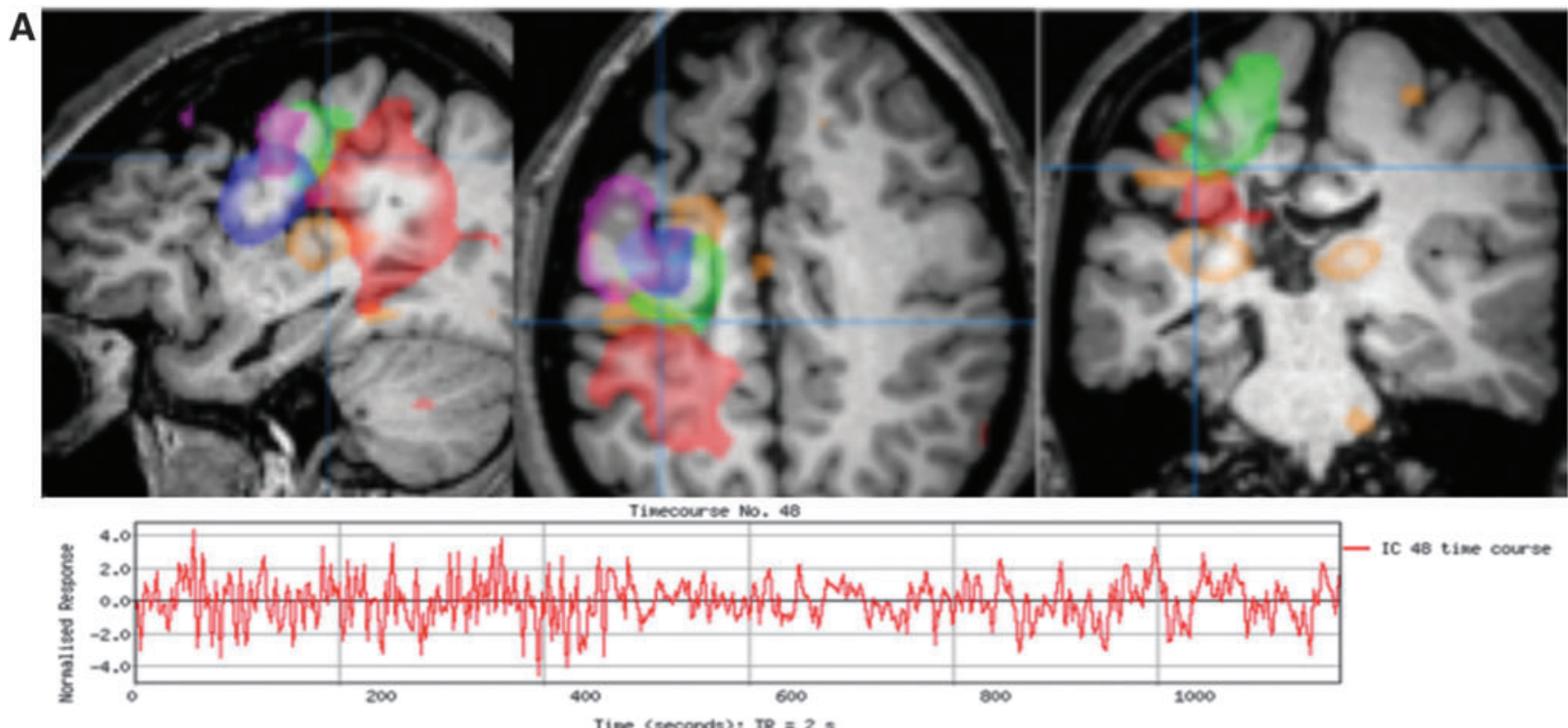

Poverspectrun of timecourse

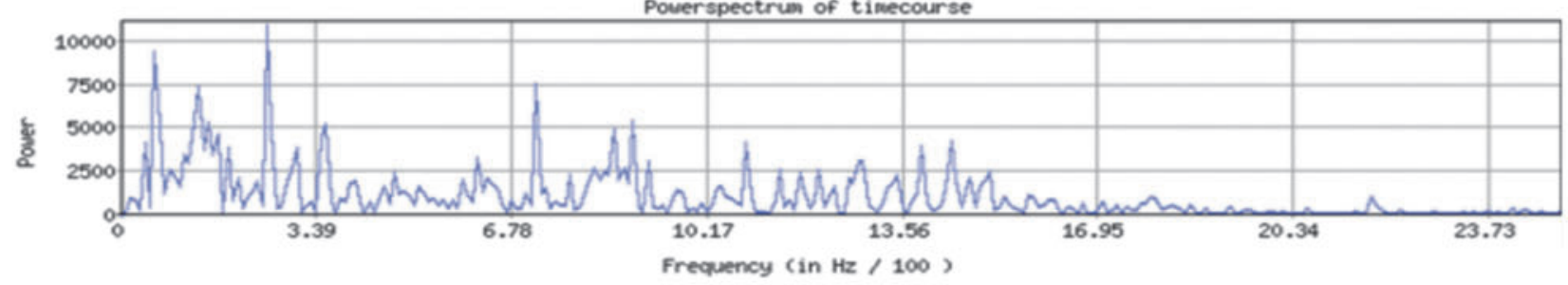

B
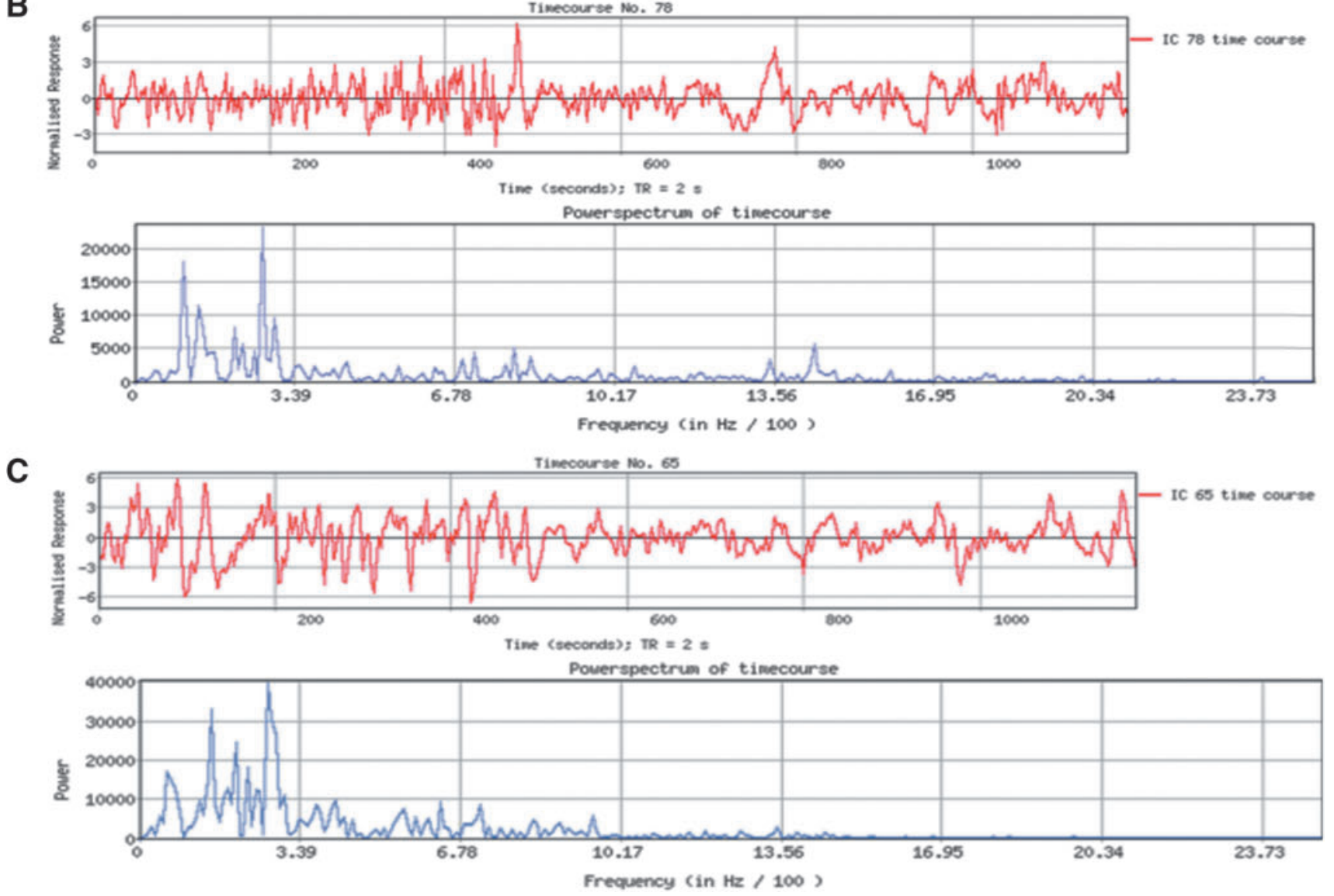

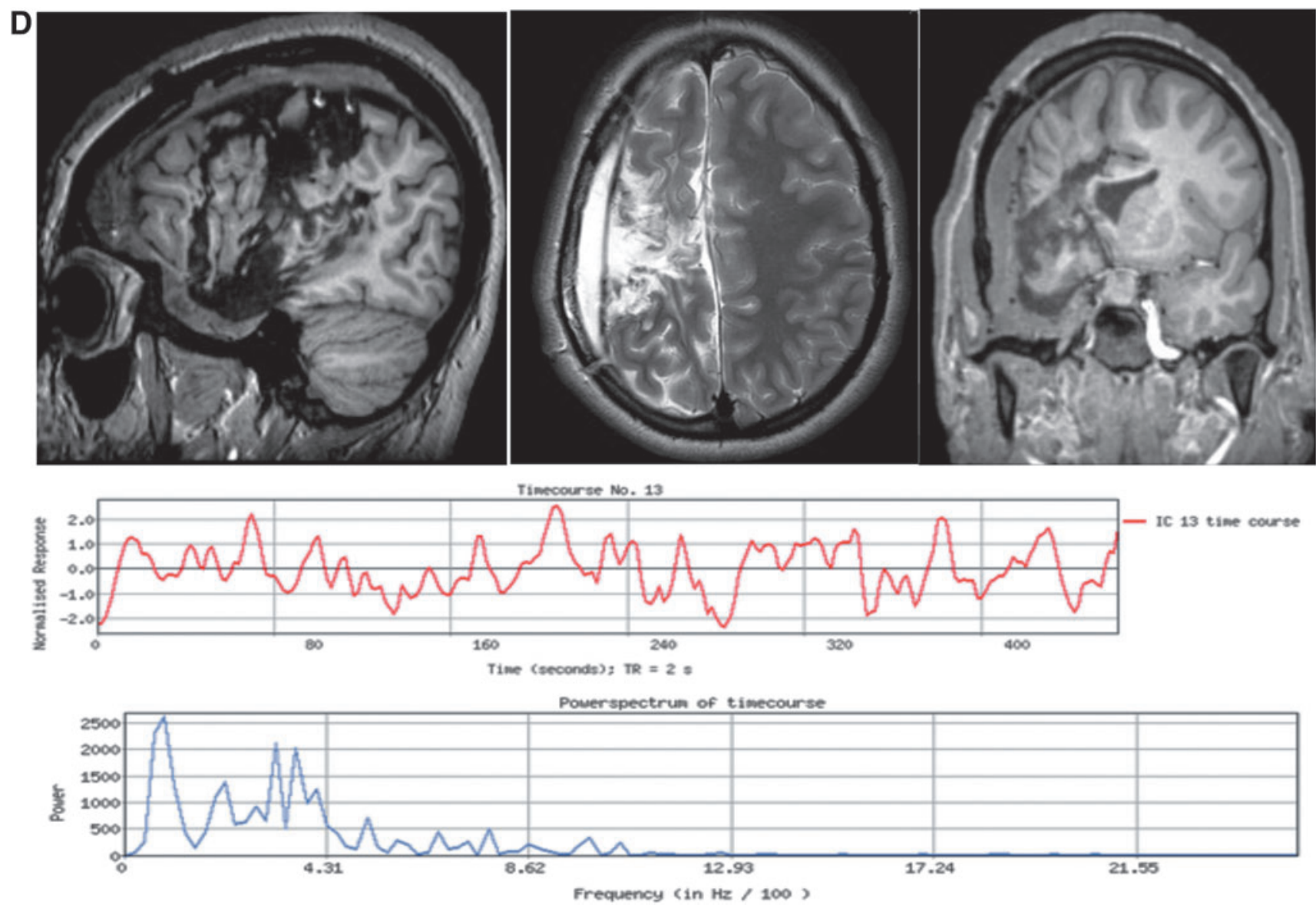

FIG. 2. (Continued).

may improve surgical planning by confirming the localization of IC-EZ to the same hemisphere or lobes as well as determining the presence of EZ outside the area planned for surgery based on other evaluative measures, apart from providing further data to the decision making for the surgical intervention and type of surgery. By grading the IC-EZs, surgeons may be better informed as to which IC-EZs are the most relevant, and whether ablation or resection of these regions would be more likely to achieve seizure control. At our center, surgeons and epileptologists determined based on the concordance of the data the extent of the IC-EZs needed to be ablated or resected by balancing the goals of decreasing seizure burden with the risk of functional deficits due to the surgery.

The findings further suggest that rs-fMRI may correlate with EZ and indicate areas to be considered for surgical destruction, since $77 \%$ remained seizure free when the entire rs-fMRI EZ was surgically removed whereas $75 \%$ continued to have seizures when a segment of rs-fMRI EZ remained after surgery. Further, this high rate of seizure recurrence when rs-fMRI EZs remained after surgery suggests that rsfMRI is a valuable tool for guiding the placement of ic-EEG to better delineate and potentially discover additional SOZ.

As illustrated, the data would suggest that since identification of an rs-fMRI EZ in a pediatric patient deemed to be a candidate for ic-EEG, it provides an opportunity that is highly specific and sensitive for diagnosis and localization before consideration for surgical intervention. Given that an rs-fMRI EZ is detected in a pediatric patient and is deemed to be a candidate for ic-EEG, there is a high $79 \%$ likelihood that the area identified by rs-fMRI is an EZ and if not surgically intervened on the child will continue to have seizures. Conversely, $21 \%$ of rs-fMRI EZ not surgically intervened on go on to significant improvement or cure of epilepsy. This may be related to the fine distinction between propagated activity, or this may lead to a further understanding of brain network dynamics. In this population, the sensitively of rs-fMRI EZ was $93 \%$, which indicated that this is potentially a high yield test, with few patients not having a potentially significant finding.

Notably, all of the patients with detectable rs-fMRI EZ and ic-EEG had an rs-fMRI EZ that agreed with the resection area. Despite this accuracy, the authors chose to categorize the $8(22 \%)$ patients who also had an anatomically separate rs-fMRI EZ from the resection, according to the outcome of this separate rs-fMRI EZ. This artificially depresses the total number of true positives. Four $(50 \%)$ of these separate rs-fMRI EZ were determined to be false positives because of the degree of the patients' clinical improvement, with resection of the ic-EEG AOZ agreeing with rs-fMRI EZ; three (38\%) were true positives in that the child continued to have seizures that were clinically consistent with the rsfMRI EZ location; and one subject's outcome (13\%) was indeterminate for such categorization. Obviously not $100 \%$ 

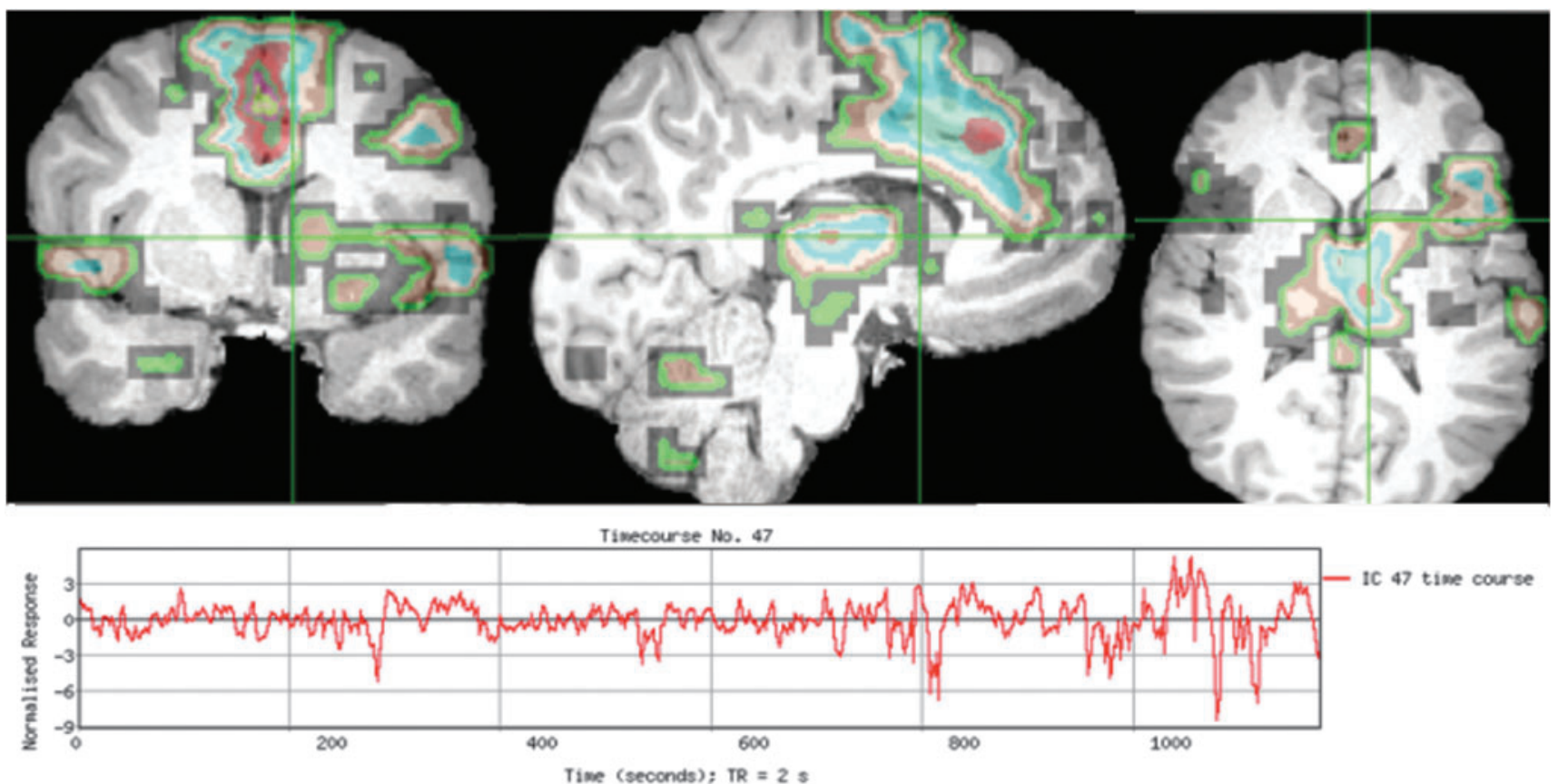

Poverspectrun of timecourse

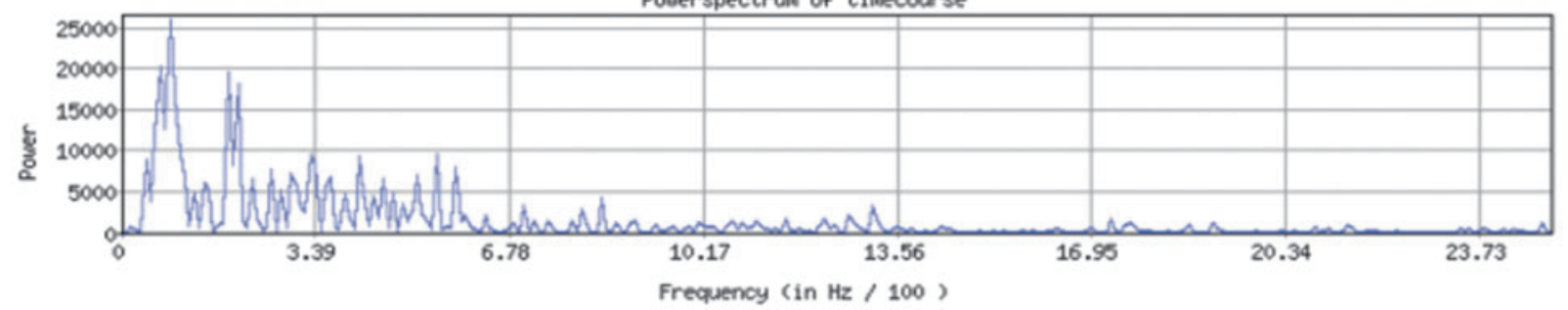

FIG. 3. Case 2: Three views of the most abnormal IC, from a patient with CLRE, which spans the left lateral, inferior, and medial frontal gyrus, into the midline supplemental motor area, and bilateral deep gray structures (more so on the left than the right). Below is the red-colored time course, showing a fast irregular spike-like oscillation pattern, and an associated blue power spectrum graph showing spread of the frequencies beyond $3.39 \mathrm{~Hz} / 100$, both of which are clearly more irregular than surrounding networks. ic-EEG covered the left prefrontal lobe, including the operculum, inferior and middle frontal gyri, medial prefrontal surface-all extending posteriorly to the supplementary sensory motor area-and into bilateral medial frontal lobes via depth electrodes. ic-EEG captured multiple seizures with consistent onset over the left lateral inferior frontal gyrus with rapid spread into left middle frontal gyrus and bilateral mesial supplementary motor areas. Resection was not performed due to this being the child's dominate expressive language center. CLRE, cryptogenic location-related epilepsy. Color images available online at www.liebertpub.com/brain

accurate, rs-fMRI still may provide insight as to the distinction between the EZ and propagated activity and provides an opportunity to further study brain network dynamics.

In this pediatric population of epilepsy surgical candidates with a broad range of seizure etiology, rs-fMRI EZ localization had a statistically significant positive predictive value of $79 \%(p<0.01)$, which is comparable to similar postoperative magnetoencephalogram results (Murakami et al., 2016).

\section{Classification and interpretation of ICs}

This study builds uniquely on prior methodology by using a data-driven approach to examine the entire brain for rs-fMRI ICs followed by a clinically driven classification paradigm to identify EZs from rs-fMRI data without the a priori guidance of ic-EEG-derived SOZs. In a prior study by Rodionov et al. (2007), ic-EEG-determined SOZ was used to confirm the location of EZs on rs-fMRI in eight patients with focal epilepsy but their algorithm did not determine whether rs-fMRI could identify EZs without ic-EEG data. This study shows that our approach can identify EZs on rs-fMRI before and independent of ic-EEG and the rs-fMRI EZs show substantial agreement with SOZs identified by ic-EEG. In addition, classifiers used by Rodionov et al. (2007) to separate noise from healthy RSNs were based on normal individuals and needed to be improved on by training with ICs that show spatial and temporal correlation with electrophysiologically confirmed SOZs (Maziero et al., 2015). However, relying on spatial correlation of an IC map to electrophysiological data (electrical source imaging or EEG) will limit the potential of the ICs to be an independent source of EZ detection. The use of rigid thresholds to remove ICs as previously done (Hunyadi et al., 2015; Zhang et al., 2015) may eliminate a number of ICs and limit the potential to localize EZs since IC-EZs can have unconstrained temporal patterns, such as frequency content both within the normal RSN range and also in higher ranges. We did not predetermine the number of ICs in each patient; the number of ICs was 

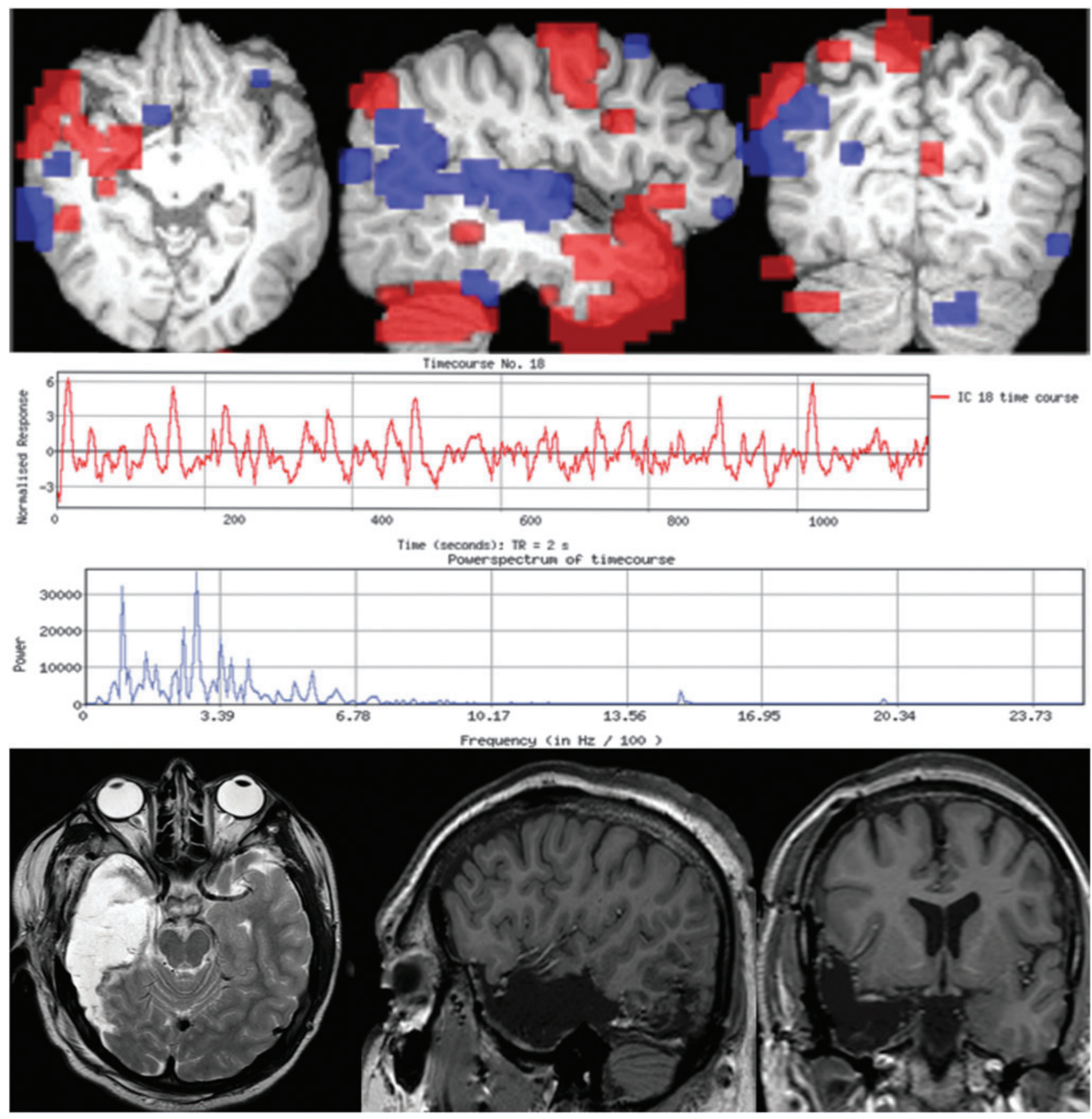

FIG. 4. Case 3: MRI images show the most abnormal IC from a patient with right mesial temporal sclerosis, where the red areas denote increased activity and the blue are the deactivated areas from the same IC, with the most spatially atypical features over the right anterior temporal pole and the hippocampus, best shown in the axial view. Unlike other abnormal ICs, this one had fairly typical temporal characteristic graphs. ic-EEG demonstrated frequent polyspikes and slow waves arising from the right superior and middle temporal gyri, and lateral greater than mesial right inferior temporal gyri. After anterior right temporal lobectomy, as shown in the bottom set of images, repeat ic-EEG was negative, and has had a substantial reduction in seizure burden with only two seizures in the past 2 years. In this case, the rs network included areas remote from the resected area, located primarily in the frontal lobe and to a lesser extent in the parietal lobe, as shown by the red areas in those lobes. Color images available online at www.liebertpub.com/brain

determined by an automated statistically driven approach with an absolute tolerance for a false discovery rate of $<0.05$. Thus, the total number of ICs was different across the subjects and supported in a consistent manner by the variance in each patient's data. This highlights a shift toward individualized analysis by using our proposed working paradigm.
Another key feature of our working paradigm to identify rs-fMRI IC-EZs is the grading of network spatial and temporal features among neuronal ICs from least to most abnormal, which we interpreted in the context of all of the other networks in that patient to maintain a whole-brain perspective. It was not unusual in this group of children with intractable 
epilepsy to have an increase in frequency from normal in nearly all networks, but the networks declared abnormal would have spatial and temporal features that are clearly more abnormal than the other networks. In almost half of the patients, more than one abnormal IC clustered in the same area. Given the grading paradigm, certain patterns were nondiagnostic, including equally abnormal globally diffuse networks. In addition, by viewing individual ICs, new findings regarding seizure propagation patterns become evident. Through ranking and comparing the abnormal ICs on an individual basis, a trend is revealed. The most abnormal sources tended to cluster spatially. After the EZ, the next highest level of atypical features tended to be in either the homologous contralateral cortex or the immediate neighboring cortex, often bleeding into local RSNs, with down-trending abnormal features as distance increased from the EZ. This contextual gradation of IC features greatly assisted with the pattern recognition of the epileptogenic focus.

\section{Comparison to other methods}

Intracranial electroencephalogram. The known caveat to ic-EEG is that only a relatively small fraction of the brain is queried, allowing conclusions only to be drawn from the studied region, and, therefore, an exclusion of other sources of seizure activity outside the studied region cannot be made. Of the nine patients with CLRE, seven went to surgery. Two of these seven had continued complete resolution of seizures 1 year postoperatively; four had a residual portion of their total SOZ removed as documented by ic-EEG, with seizure improvement $>50 \%$. This implies that in six out of the seven cases the ic-EEG correctly identified at least a portion of the true SOZ. rs-fMRI, however, is able to detect additional EZs
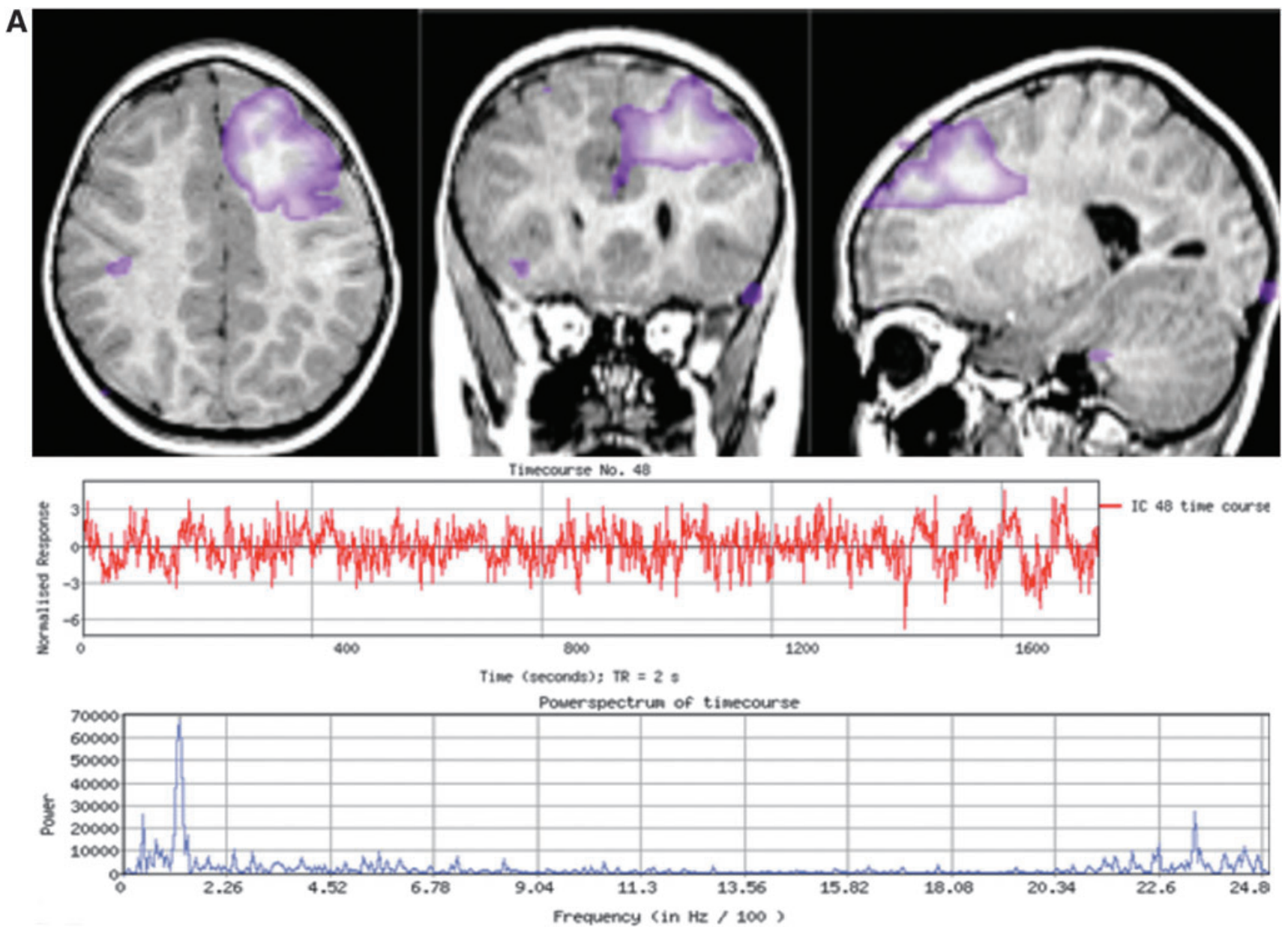

FIG. 5. Case 4: Patient with CLRE and two spatially separate abnormal ICs. (A) Left prefrontal IC with atypical spatial features spread globularly over the mesial aspect of the superior frontal gyrus and extending to the lateral edge of the middle frontal gyrus, shown with the continuously fast and erratic time course and high-frequency power spectrum. (B) Left occipital abnormal IC demonstrates equally abnormal temporal characteristics. The ic-EEG performed extensively covered the left lateral and mesial frontal and parietal lobes, as well as the right prefrontal region over superior and middle frontal gyrus. It showed nearly continuous, epileptiform activity from the left mesial greater than the lateral prefrontal lobe. Both ICs were considered abnormal and possible multifocal EZ. Corresponding left partial functional hemispherectomy and parietal lobe resection were performed, as shown in the bottom set of images. The patient's seizures were reduced, but he still has occasional seizures with visual symptoms, potentially corresponding to the second abnormal rs-fMRI ICA IC, which was not removed surgically. ICA, independent component analysis. Color images available online at www.liebertpub.com/brain 

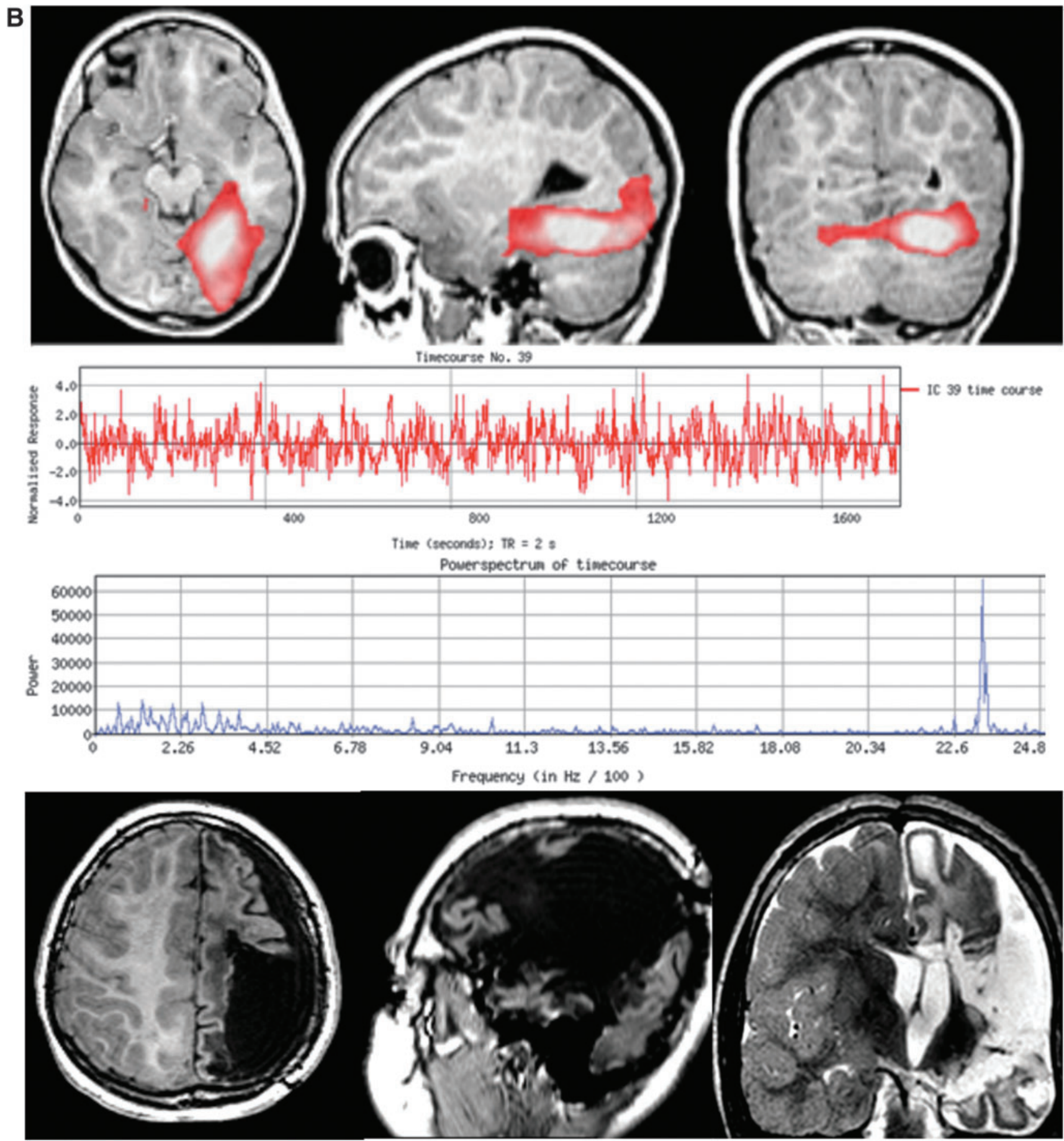

FIG. 5. (Continued).

outside the area being monitored by ic-EEG (case 4), and this may be particularly useful in planning surgical resection for patients with cryptogenic EEG, though this needs to be evaluated in a larger subgroup of these patients.

Combined EEG-fMRI. Simultaneous EEG-fMRI studies can add value to the presurgical evaluation of patients with intractable epilepsy by detecting epileptogenic foci (Dongmei et al., 2013; Thornton et al., 2010; Zijlmans et al., 2007). However, the failure rate of this test is $40-70 \%$ in adults due to lack of interictal discharges during the study and/or lack of significantly correlated hemodynamic changes (Grouiller et al., 2011). The failure rate in children may be lower due to more complex EEG data (Centeno et al., 2016) and the decreasing reliance on the detection of interictal epileptiform discharges (IEDs) during the scan (Grouiller et al., 2011). Resting-state fMRI offers advantages over EEG-fMRI since it is not event based and is less affected by variations in hemodynamic delay between different areas of the brain. Previous studies have shown evidence that IEDs are associated with 
the presence of rs-fMRI-derived markers of EZ in patients with epilepsy (Gotman and Pittau, 2011; Jackson, 2008; Maziero et al., 2015; Moeller et al., 2011; Mulert et al., 2010). As a noninvasive technique requiring minimal patient cooperation, rs-fMRI appears to be a promising tool in the identification of EZ and SOZ for the heterogenous pediatric epilepsy population.

\section{Sedation and IEDs}

Conscious sedation may be advantageous, as the average level of patient motion was $\sim 0.3 \mathrm{~mm}$ in any direction, with negligible corruption induced by patient motion. Outside these parameters, it is unknown what, if any, impact the split population of sedated and nonsedated subjects had, and from a study design standpoint uniformity in this area is theoretically superior. In a small set of patients, deep general anesthesia is clinically necessary, typically due to airway-related issues or a high amount of patient movement still occurring under light sedation. However, few, if any, networks are detectable on rs-fMRI with general anesthesia, and, therefore, this technique is not applicable to this subset of patients. Propofol, the sedative used during our rs-fMRI scans, is known to decrease the prevalence of seizures and IEDs, and it is used as a treatment in supra-refractory status epilepticus. The detection of rs-fMRI EZ in this study occurred despite the administration of propofol. Although no concurrent EEG was performed during the scan to confirm the presence of IEDs, some of the patients reported seizures close to the time of the scan. It would be reasonable to hypothesize that the effect of seizures on the brain was still occurring during the fMRI scan, and this may have been detected by the rs-fMRI EZ identified. The minimal impact of anesthesia on IEDs in this study is supported by the fact that many of these patients had IEDs during anesthesia for intraoperative ic-EEG.

\section{Limitations}

As with all commonly used EZ localization methods, expert review is required for interpretation of the clinical test results. A single clinician interpreted rs-fMRIs for 350 patients and developed the criteria that two experts applied independently to the grading of 40 pediatric epilepsy patients who underwent ic-EEG electrode placement with very high inter-rater reliability. The use of expert interpretation and small sample size are relative weaknesses of the study and method due to the degree of subjectivity in IC categorization. Also, experts had access to the anatomical images and clinical status of the patients. The experience gained yielded the working paradigm trialed as a verification process. Further objective quantification of criteria is planned with a larger patient sample size such as those modeled by De Martino et al. (2007), with the present study's modifications, taking into account the relaxation of power spectra criteria and the irregularity of distribution of BOLD signal in potential EZ ICs.

Apart from this, there was also a potential bias of the rs-fMRI reviewers to find results and assign pathological meaning as they knew that the subjects would undergo intraoperative EEG; thus, they were highly likely to have an SOZ for which an EZ might correlate. This could possible inflate sensitivity. Providing the same paradigm across a mixed population (normal, other nonepileptic pathology, epilepsy, and surgical candidates) would provide further clarification.
Lastly, in this study, a previously published optimization procedure for selecting total IC number was employed for each patient. The number of ICs computed can affect the pattern of each IC. Tasking ICA to produce more ICs could split patterns into multiple ICs. The number of ICs generated needs more analysis to determine the effect on clinical interpretation.

\section{Future directions}

In this study, we have developed a working paradigm and IC classification method by using a single investigator experience with more than 35,000 ICs in a variety of pediatric epilepsy patients. Application of this technique at other institutions is possible with physician training. The time needed to gain expertise is estimated to be similar to that of interpreting EEG. Typical rs-fMRI ICA physician time at present is $\sim 3 \mathrm{~h}$ per case, though this may decrease with further expertise and automation. The use of automated classifiers as previously described (Salimi-Khorshidi et al., 2014) may help automate some aspects of the analysis, but modification will be necessary to account for the heterogeneous nature of pediatric epilepsy patients, particularly those who are medically intractable. Building an automated IC classifier based on confirmed SOZ data for a larger, multicenter group of pediatric epilepsy patients may be best achieved through newer artificial intelligence algorithms, but it will require large data clinical data sets. Rather than choosing a finite set of quantitative image and frequency features, which do not fully reflect the individual IC contextual nature, the entire set of individual ICA data can be put through automated deeplearning algorithms with supervised outcome analysis. This would allow for learning from rich feature hierarchies to capture the complex patterns in rs-fMRI data, reduce the chance of losing information on the entire variance of the data, and offer a method to apply the working paradigm to rs-fMRI data across centers. Inclusion of healthy controls would allow a broader view of false positives - this is a part of ongoing data collection as now-healthy patients with resolved epilepsy continue to receive yearly rs-fMRIs as routine follow-up care. Future studies may also define the overlap of rs-fMRI EZ and ic-EEG SOZ that does not correspond to seizure resolution after resection.

Lastly, investigating the localization of rs-fMRI EZ in larger subgroups of epilepsy patients will help to define the unique biosignatures of rs-fMRI by epilepsy etiology. Future research will also focus on the blinding of rs-fMRI IC raters to all clinical data before interpretation, the application of conservative automated noise reduction, and techniques to augment the specificity of rs-fMRI EZ localization. Further work and comparison with other approaches to rs-fMRI analysis are needed to more fully characterize inter-rater reliability.

\section{Conclusion}

This study demonstrates the utility of the rs-fMRI EZ analysis paradigm, developed by the lead author (V.L.B.), for the preoperative evaluation of children with medically intractable epilepsy due to its substantial agreement with ic-EEGdetermined SOZs and later correlation to surgical outcomes. The finding that surgical destruction of the defined rs-fMRI EZ was highly predictive of postoperative seizure outcome highlights that rs-fMRI using this paradigm may be a useful 
tool to independently evaluate surgical epilepsy candidates, potentially identifying more surgical candidates and requiring less invasive methods for localizing the EZ.

\section{Acknowledgments}

Jeff Slavin programmed key software contributions allowing automation of the analysis. Mark Meyer of the Department of Pediatrics at Baylor College of Medicine provided editorial assistance.

\section{Author Disclosure Statement}

No competing financial interests exist.

\section{References}

Beckmann CF, DeLuca M, Devlin JT, Smith SM. 2005. Investigations into resting-state connectivity using independent component analysis. Phil Trans R Soc Lond B Biol Sci 360: 1001-1013.

Beckmann CF, Noble JA, Smith SM. 2000. Artefact detection in FMRI data using independent component analysis. Neuroimage 11:S614.

Beckmann CF, Smith SM. 2004. Probabilistic independent component analysis for functional magnetic resonance imaging. IEEE Trans Med Imaging 23:137-152.

Biswal B, Yetkin FZ, Haughton VM, Hyde JS. 1995. Functional connectivity in the motor cortex of resting human brain using echo-planar MRI. Magn Reson Med 34:537-541.

Boerwinkle VL, Wilfong AA, Curry DJ. 2016. Resting-state functional connectivity by independent component analysisbased markers corresponds to areas of initial seizure propagation established by prior modalities from the hypothalamus.

Byrt T, Bishop J, Carlin JB. 1993. Bias, prevalence and kappa. J Clin Epidemiol 46:423-429.

Centeno M, Tierney TM, Perani S, Shamshiri EA, StPier K, Wilkinson C, et al. 2016. Optimising EEG-fMRI for localisation of focal epilepsy in children. PloS One 11:e0149048.

Cordes D, Haughton VM, Arfanakis K, Carew JD, Turski PA, Moritz $\mathrm{CH}$, et al. 2001. Frequencies contributing to functional connectivity in the cerebral cortex in "resting-state" data. Am J Neuroradiol 22:1326-1333.

Damoiseaux JS, Rombouts SARB, Barkhof F, Scheltens P, Stam CJ, Smith SM, Beckmann CF. 2006. Consistent resting-state networks across healthy subjects. Proc Natl Acad Sci U S A 103:13848-13853.

De Martino F, Gentile F, Esposito F, Balsi M, Di Salle F, Goebel R, Formisano E. 2007. Classification of fMRI independent components using IC-fingerprints and support vector machine classifiers. Neuroimage 34:177-194.

Dongmei A, Fahoum F, Hall J, Olivier J, Dubeau F. 2013. Electroencephalography/functional magnetic resonance imaging responses help predict surgical outcome in focal epilepsy. Epilepsia 54:2184-2194.

Engel J. 1993. Surgical Treatment of the Epilepsies. Lippincott Williams \& Wilkins. ISBN 0-88167-988-7.

Gotman J, Pittau F. 2011. Combining EEG and fMRI in the study of epileptic discharges. Epilepsia 52(Suppl 4):38-42.

Greve DN, Fischl B. 2009. Accurate and robust brain image alignment using boundary-based registration. Neuroimage 48:63-72.

Griffanti L, Douaud G, Bijsterbosch J, Evangelisti S, AlfaroAlmagro F, Glasser MF, et al. 2017. Hand classification of fMRI ICA noise components. Neuroimage.
Grouiller F, Thornton RC, Groening K, Spinelli L, Duncan JS, Schaller K, et al. 2011. With or without spikes: Localization of focal epileptic activity by simultaneous electroencephalography and functional magnetic resonance imaging. Brain 134(Pt 10):2867-2886.

Hunyadi B, Tousseyn S, Dupont P, Van Huffel S, De Vos M, Van Paesschen W. 2015. A prospective fMRI-based technique for localizing the epileptogenic zone in presurgical evaluation of epilepsy. Neuroimage 113:329-339.

Jackson G. 2008. EEG-fMRI in the presurgical workup of patients with partial epilepsy and unclear seizure focus. Nat Clin Pract Neurol 4:128-129.

Jenkinson M, Bannister P, Brady M, Smith S. 2002. Improved optimization for the robust and accurate linear registration and motion correction of brain images. Neuroimage 17:825-841.

Jenkinson M, Smith S. 2001. A global optimisation method for robust affine registration of brain images. Med Image Anal 5:143-156.

Landis JR, Koch GG. 1977. The measurement of observer agreement for categorical data. Biometrics 33:159-174.

Lee HW, Arora J, Papademetris X, Tokoglu F, Negishi M, Scheinost D, et al. 2014. Altered functional connectivity in seizure onset zones revealed by fMRI intrinsic connectivity. Neurology 83:2269-2277.

Maziero D, Sturzbecher M, Velasco TR, Rondinoni C, Castellanos AL, Carmichael DW, Salmon CEG. 2015. A comparison of independent component analysis (ICA) of fMRI and electrical source imaging (ESI) in focal epilepsy reveals misclassification using a classifier. Brain Topogr 28:813-831.

Moeller F, LeVan P, Gotman J. 2011. Independent component analysis (ICA) of generalized spike wave discharges in fMRI: Comparison with general linear model-based EEGfMRI. Hum Brain Mapp 32:209-217.

Mulert C, Leicht G, Hepp P, Kirsch V, Karch S, Pogarell O, et al. 2010. Single-trial coupling of the gamma-band response and the corresponding BOLD signal. Neuroimage 49:2238-2247.

Murakami H, Wang ZI, Marashly A, Krishnan B, Prayson RA, Kakisaka Y, et al. 2016. Correlating magnetoencephalography to stereo-electroencephalography in patients undergoing epilepsy surgery. Brain 139:2935-2947.

Negishi M, Martuzzi R, Novotny EJ, Spencer DD, Constable RT. 2011. Functional MRI connectivity as a predictor of the surgical outcome of epilepsy. Epilepsia 52:1733-1740.

Pruim RHR, Mennes M, van Rooij D, Llera A, Buitelaar JK, Beckmann CF. 2015. ICA-AROMA: A robust ICA-based strategy for removing motion artifacts from fMRI data. Neuroimage 112:267-277.

Rodionov R, De Martino F, Laufs H, Carmichael DW, Formisano E, Walker M, et al. 2007. Independent component analysis of interictal fMRI in focal epilepsy: Comparison with general linear model-based EEG-correlated fMRI. Neuroimage 38:488-500.

Salimi-Khorshidi G, Douaud G, Beckmann CF, Glasser MF, Griffanti L, Smith SM. 2014. Automatic denoising of functional MRI data: Combining independent component analysis and heirarchical fusion of classifiers. Neuroimage 90:449-468.

Schrouff J, Perlbarg V, Boly M, Marrelec G, Boveroux P, Vanhaudenhuyse A, et al. 2011. Brain functional integration decreases during propofol-induced loss of consciousness. Neuroimage 57:198-205.

Smith SM, Fox PT, Miller KL, Glahn DC, Fox PM, Mackay $\mathrm{CE}$, et al. 2009. Correspondence of the brain's functional architecture during activation and rest. Proc Natl Acad Sci U S A 106:13040-13045. 
Stufflebeam SM, Liu H, Sepulcre J, Tanaka N, Buckner RL, Madsen JR. 2011. Localization of focal epileptic discharges using functional connectivity magnetic resonance imaging: Clinical article. J Neurosurg 114:1693-1697.

Thornton RC, Rodionov R, Laufs H, Vulliemoz S, Vaudano A, Carmichael D, et al. 2010. Imaging haemodynamic changes related to seizures: Comparison of EEG-based general linear model, independent component analysis of fMRI and intracranial EEG. Neuroimage 53:196-205.

Vanderby SA, Babyn PS, Carter MW, Jewell SM, McKeever PD. 2010. Effect of anesthesia and sedation on pediatric MR imaging patient flow. Radiology 256:229-237.

Zhang CH, Lu Y, Brinkmann B, Welker K, Worrell G, He B. 2014. Using functional MRI alone for localization in focal epilepsy. Conf Proc IEEE Eng Med Biol Soc 2014:730-733.

Zhang CH, Lu Y, Brinkmann B, Welker K, Worrell G, He B. 2015. Lateralization and localization of epilepsy related he- modynamic foci using presurgical fMRI. Clin Neurophysiol 126:27-38.

Zijlmans M, Huiskamp G, Hersevoort M, Seppenwoolde JH, van Huffelen AC, Leijten FSS. 2007. EEG-fMRI in the preoperative work-up for epilepsy surgery. Brain 130: 2343-2353.

Address correspondence to: Varina L. Boerwinkle Division of Pediatric Neurology Barrow Neurological Institute at Phoenix Children's Hospital 1919 E. Thomas Road Ambulatory Building, Floor 3 Phoenix, AZ, 85016

E-mail: vboerwinkle@phoenixchildrens.com 